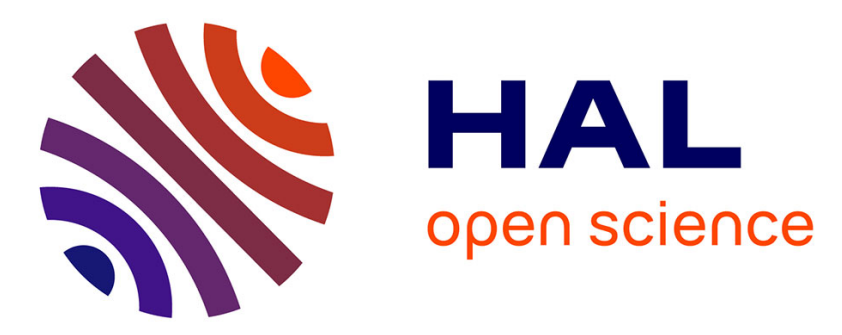

\title{
An Explicit Isometric Reduction of the Unit Sphere into an Arbitrarily Small Ball
}

Evangelis Bartzos, Vincent Borrelli, Roland Denis, Francis Lazarus, Damien Rohmer, Boris Thibert

\section{- To cite this version:}

Evangelis Bartzos, Vincent Borrelli, Roland Denis, Francis Lazarus, Damien Rohmer, et al.. An Explicit Isometric Reduction of the Unit Sphere into an Arbitrarily Small Ball. Foundations of Computational Mathematics, 2018, 18 (4), pp.1015-1042. 10.1007/s10208-017-9360-1 . hal-01647062

\section{HAL Id: hal-01647062 \\ https://hal.inria.fr/hal-01647062}

Submitted on 24 Nov 2017

HAL is a multi-disciplinary open access archive for the deposit and dissemination of scientific research documents, whether they are published or not. The documents may come from teaching and research institutions in France or abroad, or from public or private research centers.
L'archive ouverte pluridisciplinaire HAL, est destinée au dépôt et à la diffusion de documents scientifiques de niveau recherche, publiés ou non, émanant des établissements d'enseignement et de recherche français ou étrangers, des laboratoires publics ou privés. 


\title{
AN EXPLICIT ISOMETRIC REDUCTION OF THE UNIT SPHERE INTO AN ARBITRARILY SMALL BALL
}

\author{
DEDICATED TO THE MEMORY OF DAVID SPRING
}

\author{
E. BARTZOS, V. BORRELLI, R. DENIS, F. LAZARUS, D. ROHMER, AND B. THIBERT
}

\begin{abstract}
Spheres are known to be rigid geometric objects: they cannot be deformed isometrically, i.e. while preserving the length of curves, in a twice differentiable way. An unexpected result by J. Nash (Ann. of Math. 60: 383-396, 1954) and N. Kuiper (Indag. Math. 17: 545-555, 1955) shows that this is no longer the case if one requires the deformations to be only continuously differentiable. A remarkable consequence of their result makes possible the isometric reduction of a unit sphere inside an arbitrarily small ball. In particular, if one views the Earth as a round sphere the theory allows to reduce its diameter to that of a terrestrial globe while preserving geodesic distances.

Here we describe the first explicit construction and visualization of such a reduced sphere. The construction amounts to solve a non-linear PDE with boundary conditions. The resulting surface consists of two unit spherical caps joined by a $C^{1}$ fractal equatorial belt. An intriguing question then arises about the transition between the smooth and the $C^{1}$ fractal geometries. We show that this transition is similar to the one observed when connecting a Koch curve to a line segment.
\end{abstract}

\section{INTRODUCTION}

Mathematics is an inexhaustible source of strange and extraordinary objects whose visualization can be quite challenging. Among others we may cite the visualization of the projective plane $[6,1]$, of fractals or limit sets $[16,17]$, or of the sphere eversion $[15,10]$. Each of these visualizations brought new ideas and unveiled properties that were hardly conceivable before. The realization of an immersed projective plane by W. Boy was a visual answer to an erroneous supposition of D. Hilbert. The search for an intelligible visualization of the sphere eversion led W. Thurston to the discovery of the Theory of Corrugations, a qualitative version of convex integration. The visualization of fractals has changed our perception of the world and deepened the mathematical representation of nature.

Here we achieve the explicit construction and the first visualization of a paradoxical object whose formal existence was proved in the mid-fifties by J. Nash and N. Kuiper [18, 14]: an isometric reduced sphere. The existence of such a surface is in sharp contrast with the rigidity of round spheres: every $C^{2}$ map that preserves distances over the round unit sphere is the restriction of a rigid motion [7, 12]. However, the works of J. Nash and N. Kuiper shows that this rigidity breaks down when the map is of regularity $C^{1}$. This result was recently extended to $C^{1, \alpha}$ maps for some $\alpha>0$ by Conti et al. [8] (see also Borisov [2,3]). In this range of

Date: July 7, 2017.

1991 Mathematics Subject Classification. Primary 35-04, 53C21; Secondary 53C42.

This work is part of the HeveA project and was partly supported by the LabEx PersYval-LaB ANR-11LABX-0025-01. The first author was in internship at the Institut CAMILlE Jordan. The third author was a post-doc financed by the Matstic grant First from University Joseph Fourier and by Laboratoire Jean Kuntzmann. We are also thankful to the Grenoble University High Performance Computing Centre project (CIMENT) for providing access to its computing platform. 
regularity, round spheres become flexible and can be reduced, i.e. sent isometrically inside a ball of smaller radius.

Our approach relies on the theory of convex integration developed by Gromov [11, 19, 9]. It fills the gap between this deep piece of mathematics and explicit constructions amenable to computation. We reduce a sphere isometrically by cutting out two antipodal caps of a round unit sphere, bringing them closer and compressing the remaining equatorial belt to fit in-between the boundaries of the caps. Although the translation of the caps is isometric, it is far from obvious that the compression can be done isometrically and even less obvious that the resulting belt connects continuously with the caps. This is accomplished by adapting the theory of convex integration with a global point of view that avoids piecing together local processes.

In $[4,5]$ we build an isometric embedding of a flat torus and describe a new geometric structure halfway between smooth surfaces and fractals that we have called $C^{1}$-fractal. In this paper we also address the question of how to connect the $C^{1}$-fractal structure of the compressed equatorial belt with the smooth caps to construct a $C^{1}$ isometric reduced sphere. This connection amounts to consider boundary conditions in the theory of convex integration. Although this theory formally allows boundary conditions, its effective application and the understanding of the resulting geometry remains a crucial issue for the implementation of convex integration as an efficient procedure for solving partial differential relations.

In order to handle the boundary conditions, we introduce a phase shift and non-integral corrugation numbers in the Convex Integration Formula as well as intermediate differential relations defined over an increasing sequence of equatorial ribbons. It produces a sequence of corrugated surfaces that $C^{1}$ converges towards an isometric reduced sphere. Based on this implementation we obtain the first rendered images of an isometric embedding of a unit sphere inside a small ball. If one views the Earth as a round sphere the procedure makes it possible to reduce its diameter to that of a terrestrial globe while preserving geodesic distances. Due to numerical reasons and visual limitations, we chose to reduce the size by a factor of two. As a matter of fact, the resulting geometry exhibits the same structure independently of the reduction factor: the Gauss map of the equatorial belt can be expressed as the remainder of an infinite product that fades out as we get closer to the boundary of the caps. Moving from one of the caps toward the equator, corrugations of larger and larger amplitudes emerge creating what we have called a $C^{1}$ fractal expansion. See Theorem 13 and Figure 1. This remarkable phenomenon explains the transition between the analytic regularity of the caps and the $C^{1}$ regularity of the equatorial belt analogous to the transition between a line segment and a von Koch curve. See Figure 10.

This paper is organized as follows. Our construction of a reduced isometric sphere starts with an initial embedding that shortens distances as described in Section 2. After recalling the Convex Integration process for curves, we describe in Section 4 how we extend this process to surfaces with boundary conditions. We apply this two dimensional process to the equatorial belt of our initial embedding. We discuss in Section 5 how far is the resulting embedding from an isometric one. The Convex Integration process must be applied iteratively as explained in Section 6, where we provide all the ingredients to construct a reduced isometric sphere. The proof of convergence of the iterative process is the object of Sections 7 and 8. The transition between the analytic caps and the $C^{1}$ equatorial belt is examined in Section 9. This analysis reveals the $C^{1}$ fractal expansion. 

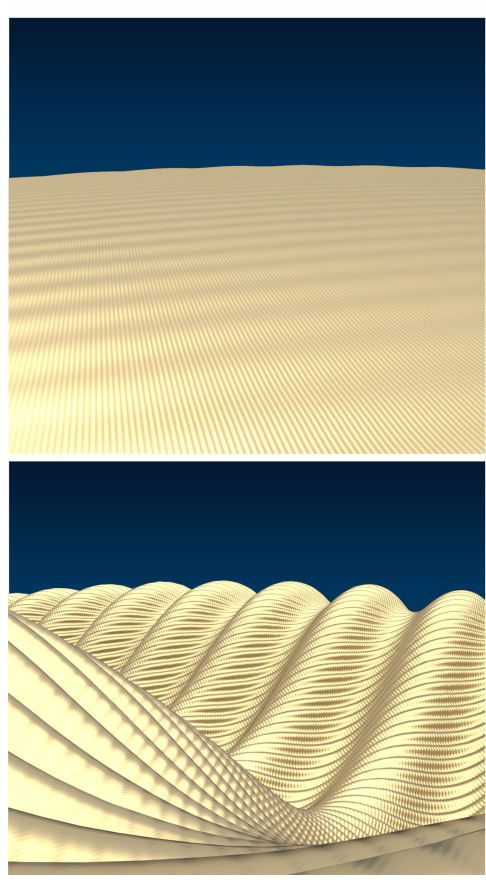
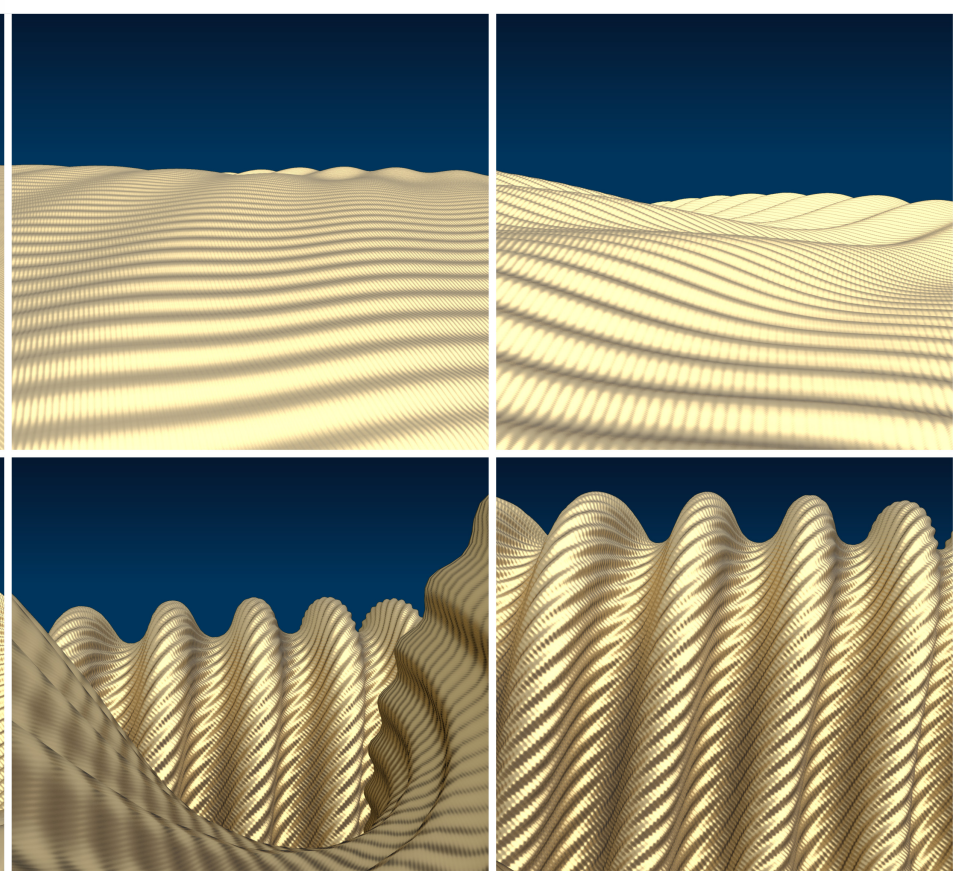

FiguRE 1. A walk from the boundary of the south polar cap to the equatorial belt showing the $C^{1}$ fractal expansion.

\section{The Initial MaP}

The construction of a $C^{1}$ isometric reduced sphere ${ }^{1}$ starts with an initial map $f_{0}: \mathbb{S}^{2} \rightarrow$ $B_{r} \subset \mathbb{R}^{3}$ where $B_{r}$ is a ball of radius $r<1$ centered at the origin and $\mathbb{S}^{2}$ denotes the unit sphere. In this section, we describe sufficient conditions for the initial map $f_{0}$ to allow our convex integration process to handle boundary conditions. We first cut $\mathbb{S}^{2}$ into three parts: two small spherical caps centered at the poles and a complementary equatorial belt $\mathcal{B} \subset \mathbb{S}^{2}$. Here, the caps are considered as closed sets so that $\mathcal{B}$ is open in $\mathbb{S}^{2}$. The map $f_{0}$ vertically translates each cap into $B_{r}$. The restriction of $f_{0}$ to the equatorial belt should satisfy the following conditions.

(C1) $\quad f_{\left.0\right|_{\mathcal{B}}}$ has a $C^{\infty}$ regularity,

(C2) $\quad f_{0}(\mathcal{B})$ is included in $B_{r}$,

(C3) $f_{\left.0\right|_{\mathcal{B}}}$ connects the two polar caps with a $C^{1}$ regularity.

We also require a loop condition imposed by the technique of convex integration applied to the construction of isometric maps. In order to express this condition we need some settings. We shall denote by $S_{0}:=f_{0}\left(\mathbb{S}^{2}\right)$ the initial surface and by $R_{0}:=f_{0}(\mathcal{B})$ the shrunk central ribbon. See Figure 2 for an illustration. We also consider the usual parameterization of the sphere:

$$
h:(x, y) \mapsto(\cos y \cos x, \cos y \sin x, \sin y)
$$

\footnotetext{
${ }^{1}$ Very recently, a formal construction of a deformed isometric sphere was obtained by considering isometric extensions [13, Cor. 1.3]. However, one equator is left unchanged in this approach, which prevents the sphere to be globally reduced.
} 


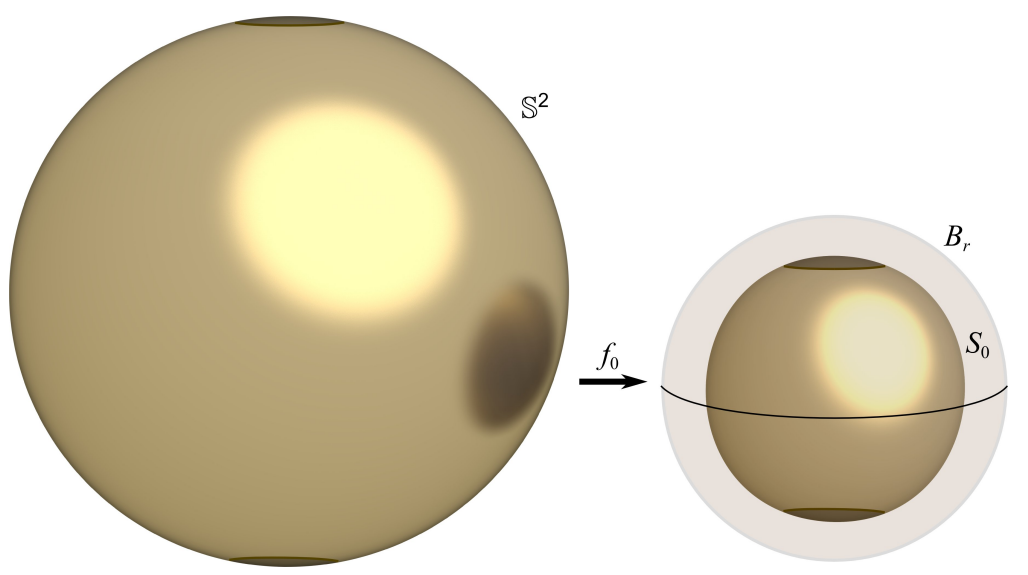

FiguRE 2. The map $f_{0}: \mathbb{S}^{2} \rightarrow B_{r} \subset \mathbb{R}^{3}$. The shrunk central ribbon $R_{0}$ is a surface of revolution generated by rotating a polynomial curve to satisfy Conditions $(\mathrm{C} 1)-(\mathrm{C} 4)$. The initial isometric error is $E\left(f_{0}\right) \approx 1.17$.

We introduce the parameter domain $\left.\mathcal{D}:=\mathbb{S}^{1} \times\right]-y_{\infty}, y_{\infty}\left[\right.$ where $\mathbb{S}^{1}=\mathbb{R} / 2 \pi \mathbb{Z}$ and $y_{\infty}$ is the polar angle of the upper cap boundary. We denote by $\widetilde{\mathcal{D}}:=\mathbb{R} \times]-y_{\infty}, y_{\infty}[$ its universal cover. Remark that $h(\mathcal{D})$ is the equatorial belt $\mathcal{B}$. With a little abuse of notation we still denote by $f_{0}: \mathcal{D} \rightarrow R_{0}$ the map defining the central ribbon. We denote by $I_{f_{0}}$ and $I_{h}$ the first fundamental forms of the maps $f_{0}$ and $h$. Using the same notation for a bilinear form and its matrix, we thus have

$$
I_{f_{0}}=\left(\begin{array}{ll}
\left\langle\frac{\partial f_{0}}{\partial x}, \frac{\partial f_{0}}{\partial x}\right\rangle & \left\langle\frac{\partial f_{0}}{\partial x}, \frac{\partial f_{0}}{\partial y}\right\rangle \\
\left\langle\frac{\partial f_{0}}{\partial x}, \frac{\partial f_{0}}{\partial y}\right\rangle & \left\langle\frac{\partial f_{0}}{\partial y}, \frac{\partial f_{0}}{\partial y}\right\rangle
\end{array}\right) \quad \text { and } \quad I_{h}=\left(\begin{array}{cc}
\cos ^{2} y & 0 \\
0 & 1
\end{array}\right)
$$

where $\langle\cdot, \cdot\rangle$ is the usual inner product. We next introduce the following constant linear forms in $\widetilde{\mathcal{D}}$ :

$$
\ell_{1}(w)=\frac{1}{\sqrt{2}}(X+Y), \quad \ell_{2}(w)=\frac{1}{\sqrt{2}}(Y-X), \quad \ell_{3}(w)=Y
$$

where $w=X e_{X}+Y e_{Y}$ and $\left(e_{X}, e_{Y}\right)$ is the canonical basis of $\mathbb{R}^{2}$. We shall use the same notation $\ell_{i}$ for the field of linear forms in $\widetilde{\mathcal{D}}$ and its quotient in $\mathcal{D}$. We denote by $\ell_{i} \otimes \ell_{i}$ the degenerate bilinear form $\left(w, w^{\prime}\right) \mapsto \ell_{i}(w) \ell_{i}\left(w^{\prime}\right)$, also called a primitive metric. Since any symmetric bilinear form can be uniquely written as a linear combination of the $\ell_{i} \otimes \ell_{i}$ s, we have at every point $(x, y)$ :

$$
I_{h}-I_{f_{0}}=\sum_{i=1}^{3} \rho_{i}\left(I_{h}-I_{f_{0}}\right) \ell_{i} \otimes \ell_{i}
$$

We call the real valued functions $\rho_{i}\left(I_{h}-I_{f_{0}}\right): \mathcal{D} \rightarrow \mathbb{R}$ the primitive coordinates of the bilinear form $I_{h}-I_{f_{0}}$ with respect to the $\ell_{i} \otimes \ell_{i}$ s. Note that for any symmetric bilinear form $B$, we easily show that

$$
\left|\rho_{1}(B)\right|,\left|\rho_{2}(B)\right| \leqslant \frac{\sqrt{6}}{2}\|B\| \text { and }\left|\rho_{3}(B)\right| \leqslant \sqrt{2}\|B\|
$$

Here and from now on, $\|\cdot\|$ denotes the Frobenius norm when applied to matrices. We can now express the loop condition for the pair $\left(f_{0}, h\right)$ : 
AN EXPLICIT ISOMETRIC REDUCTION OF THE UNIT SPHERE INTO AN ARBITRARILY SMALL BALLDEDICATED TO THE MEMO

The primitive coordinates of $I_{h}-I_{f_{0}}$ are strictly positive over $\mathcal{D}$.

This last condition implies that $f_{0}$ is strictly short over $\mathcal{D}$, i.e. for every non-zero vector $w$ we have $w^{t} I_{h} w>w^{t} I_{f_{0}} w$. As a consequence, for every curve $\gamma:[0,1] \rightarrow \mathcal{D}$, the length of $f_{0} \circ \gamma$ is strictly less than the length of $h \circ \gamma$. It will also be convenient (see Lemma 12) to assume that the skew diagonal coefficients of $I_{f_{0}}$ cancel everywhere. The next proposition states that this is always possible.

Proposition 1. There exists an initial map $f_{0}: \mathcal{D} \rightarrow \mathbb{R}^{3}$ that satisfies Conditions (C1)-(C4). Moreover, $f_{0}$ can be chosen with a symmetry of revolution, i.e., of the form

$$
f_{0}(x, y)=(X(y) \cos (x), X(y) \sin (x), Z(y))
$$

where $(X, Z):\left[-y_{\infty}, y_{\infty}\right] \rightarrow \mathbb{R}^{2}$ is a $C^{\infty}$ profile curve. In particular, $\left\langle\frac{\partial f_{0}}{\partial x}, \frac{\partial f_{0}}{\partial y}\right\rangle \equiv 0$.

Our goal is to produce an isometric image of the unit sphere that lies inside the ball $B_{r}$ of radius $r<1$. This amounts to replace $f_{0}$ by a map $f: \mathcal{D} \rightarrow \mathbb{R}^{3}$ such that the new central ribbon $f(\mathcal{D})$

i) lies inside the ball $B_{r}$,

ii) connects the two caps of $S_{0}$ with a $C^{1}$ regularity,

iii) is isometric to $\mathcal{B}$.

Indeed, since the caps are isometric to their copies in $\mathbb{S}^{2}$, we only need to make the central ribbon isometric to $\mathcal{B}$. This will be the case if $f$ and $h$ have the same first fundamental forms, i.e. $I_{f}=I_{h}$. We call $E\left(f_{0}\right)=\sup _{\mathcal{D}}\left\|I_{h}-I_{f_{0}}\right\|$ the isometric error. Hence, $f$ is an isometric map if and only if $E(f)=0$.

\section{Proof of Proposition 1}

Here we prove the existence of a profile curve $\gamma=(X, Z):\left[-y_{\infty}, y_{\infty}\right] \rightarrow \mathbb{R}^{2}$ as in the proposition. After writing down the conditions that $\gamma$ should satisfy, we construct a suitable $\gamma$ in three steps. We first cut the profile curve into three pieces and provide explicit formulas for each piece. The resulting curve is piecewise $C^{\infty}$ with continuous connections and satisfies the conditions on each piece. We next apply a convex integration process to the center piece in order to get a $C^{1}$ connection. We finally obtain a $C^{\infty}$ profile curve by regularization.

Let $y_{\infty}$ and $\eta$ be such that

$$
\cos ^{2} y_{\infty}+\left(\sin y_{\infty}-\eta\right)^{2}<r^{2} \quad \text { and } \quad \frac{\sin y_{\infty}-\eta}{y_{\infty}}<\cos y_{\infty}
$$

The first inequality ensures that the vertical translations of the two caps by $\pm \eta$ are contained in $B_{r}$. The reason for the second inequality will appear below. A simple calculation shows that

$$
I_{h}-I_{f_{0}}=\left(\begin{array}{cc}
\cos ^{2} y-X^{2}(y) & 0 \\
0 & 1-X^{\prime 2}(y)-Z^{\prime 2}(y)
\end{array}\right)
$$

and thus $\rho_{1}\left(I_{h}-I_{f_{0}}\right)=\cos ^{2} y-X^{2}(y), \rho_{2}\left(I_{h}-I_{f_{0}}\right)=\cos ^{2} y-X^{2}(y)$ and $\rho_{3}\left(I_{h}-I_{f_{0}}\right)=$ $\sin ^{2} y+X^{2}(y)-X^{\prime 2}(y)-Z^{\prime 2}(y)$. Hence Conditions (C2) and (C4) are equivalent to

$$
\left\{\begin{array}{l}
\cos ^{2} y \geqslant X^{2}(y) \geqslant v^{2}(y)-\sin ^{2} y \quad \text { where } v^{2}=X^{\prime 2}(y)+Z^{\prime 2}(y) . \\
X^{2}+Z^{2}<r^{2}
\end{array}\right.
$$


Condition (C3) is equivalent to

$$
\left\{\begin{array} { l } 
{ X ( - y _ { \infty } ) = \operatorname { c o s } y _ { \infty } } \\
{ Z ( - y _ { \infty } ) = \eta - \operatorname { s i n } ( y _ { \infty } ) } \\
{ X ^ { \prime } ( - y _ { \infty } ) = \operatorname { s i n } y _ { \infty } } \\
{ Z ^ { \prime } ( - y _ { \infty } ) = \operatorname { c o s } y _ { \infty } . }
\end{array} \quad \text { and } \quad ( 3 . 7 ) \quad \left\{\begin{array}{l}
X\left(y_{\infty}\right)=\cos y_{\infty} \\
Z\left(y_{\infty}\right)=\sin \left(y_{\infty}\right)-\eta \\
X^{\prime}\left(y_{\infty}\right)=-\sin y_{\infty} \\
Z^{\prime}\left(y_{\infty}\right)=\cos y_{\infty} .
\end{array}\right.\right.
$$

Step 1. A Taylor expansion at $-y_{\infty}$ implies that for some small enough $\delta>0$ the piece of parabola $\gamma_{1}:\left[-y_{\infty}, \delta-y_{\infty}\right] \rightarrow \mathbb{R}^{2}$ defined by

$$
\left\{\begin{array}{l}
X(y)=\cos y_{\infty}+\left(\sin y_{\infty}\right)\left(y+y_{\infty}\right)-\left(\cos y_{\infty}\right)\left(y+y_{\infty}\right)^{2} \\
Z(y)=\eta-\sin y_{\infty}+\left(\cos y_{\infty}\right)\left(y+y_{\infty}\right)
\end{array}\right.
$$

satisfies Equations (3.5) and (3.6). A symmetric formula holds on the interval $\left[y_{\infty}-\delta, y_{\infty}\right]$ leading to a curve $\gamma_{3}:\left[y_{\infty}-\delta, y_{\infty}\right] \rightarrow \mathbb{R}^{2}$ satisfying Equations (3.5) and (3.7). We then define $\gamma_{2}$ over the interval $I_{\delta}:=\left[-\left(y_{\infty}-\delta\right), y_{\infty}-\delta\right]$ as the linear interpolation between $\gamma_{1}\left(\delta-y_{\infty}\right)$ and $\gamma_{3}\left(y_{\infty}-\delta\right)$. This vertical line segment connects continuously with $\gamma_{1}$ and $\gamma_{3}$. Furthermore, choosing $\delta$ smaller if needed, and using the second inequality in the above condition (3.4) we easily infer that $\gamma_{2}$ satisfies Equation (3.5). We denote by $X_{\delta}:=\cos y_{\infty}+\left(\sin y_{\infty}\right) \delta-\left(\cos y_{\infty}\right) \delta^{2}$ the constant abscissa of $\gamma_{2}$.

Step 2. In order to obtain a $C^{1}$ connection at $\gamma_{1}\left(\delta-y_{\infty}\right)$ and $\gamma_{3}\left(y_{\infty}-\delta\right)$ we shall use Lemma 17.3.1 in [9]. To this end we view Equation (3.5) as the differential relation

$$
\mathcal{R}=\left\{(y, X, Z, \mathcal{U}, \mathcal{V}) \in \mathbb{R} \times \mathbb{R}^{2} \times \mathbb{R}^{2}, \cos ^{2} y \geqslant X^{2} \geqslant \mathcal{U}^{2}+\mathcal{V}^{2}-\sin ^{2} y \text { and } X^{2}+Z^{2}<r^{2}\right\} .
$$

We next consider a continuous interpolation $\psi_{2}=(\mathcal{U}, \mathcal{V}): I_{\delta} \rightarrow \mathbb{R}^{2}$ between $\psi_{2}\left(\delta-y_{\infty}\right):=$ $\gamma_{1}^{\prime}\left(\delta-y_{\infty}\right)$ and $\psi_{2}\left(y_{\infty}-\delta\right):=\gamma_{3}^{\prime}\left(y_{\infty}-\delta\right)$. Up to renormalization we can assume that $\left\|\psi_{2}(y)\right\|^{2}<$ $X_{\delta}^{2}+\sin ^{2} y$ for all $y \in I_{\delta}$. It follows that $\left(\gamma_{2}, \psi_{2}\right)$ is a short formal solution of $\mathcal{R}$. According to Lemma 17.3.1 in [9], we can construct a genuine solution $\tilde{\gamma}_{2}$ of $\mathcal{R}$ such that its derivative coincides with $\psi_{2}$ at $\delta-y_{\infty}$ and $y_{\infty}-\delta$. The concatenation $\gamma$ of $\gamma_{1}, \tilde{\gamma}_{2}$ and $\gamma_{3}$ is a $C^{1}$ curve that fulfills Conditions (3.5), (3.6) and (3.7).

Step 3. We finally apply some smoothing to $\gamma$ to obtain a $C^{\infty}$ curve that still satisfies Conditions (3.5), (3.6) and (3.7). The corresponding map $f_{0}$ meets all the requirements (C1)(C4).

In practice we have chosen for $\gamma$ a single Hermite cubic spline re-parametrized by another Hermite cubic spline. The resulting profile curve has polynomial coordinates of degree nine and we have checked numerically Conditions (C1)-(C4). See Figure 2.

\section{Convex Integration Along Curves}

As noted above the initial map $f_{0}: \mathcal{D} \rightarrow \mathbb{R}^{3}$ shortens distances, implying that its isometric error is positive. Convex integration is a tool to reduce the isometric error by elongating a family of curves. In this section, we detail the Convex Integration process for a family of curves that foliates the universal cover $\widetilde{\mathcal{D}}$ of $\mathcal{D}$. We first recall the Convex Integration Formula for one curve established in [5]. This formula does not adapt to fit the boundary conditions. To take them into account we introduce a phase shift and use non-integral corrugation numbers (see Formula (4.10) and Lemma 2). The new formula creates a map $\widetilde{\mathcal{D}} \rightarrow \mathbb{R}^{3}$ that descends to the quotient. We then show how to control its transverse derivative in Lemma 3.

Convex Integration for one curve. Given a curve $\gamma:[0,1] \rightarrow \mathcal{D}$ and a target speed function $r:[0,1] \rightarrow \mathbb{R}_{>0}$ greater than the speed function of $f_{0} \circ \gamma$, i.e. for all $s \in[0,1]$, 
AN EXPLICIT ISOMETRIC REDUCTION OF THE UNIT SPHERE INTO AN ARBITRARILY SMALL BALLDEDICATED TO THE MEMO

$\left\|\left(f_{0} \circ \gamma\right)^{\prime}(s)\right\| \leqslant r(s)$, we shall construct a new curve $\Gamma$ close to $f_{0} \circ \gamma$ such that $\left\|\Gamma^{\prime}(s)\right\|=r(s)$. We consider the following formula whose origin stems from Convex Integration Theory [5]:

$$
s \mapsto \Gamma(s):=f_{0}(\gamma(0))+\int_{0}^{s} r(\sigma)(\cos \theta \mathbf{t}(\gamma(\sigma))+\sin \theta \mathbf{n}(\gamma(\sigma))) \mathrm{d} \sigma
$$

where $\mathbf{t}(\gamma(\sigma))$ is the unit vector tangent to the curve $f_{0} \circ \gamma$ and $\mathbf{n}(\gamma(\sigma))$ is the normal to $S_{0}$ at the point $f_{0} \circ \gamma(\sigma)$. Here, $\theta:=\theta(\sigma)=\alpha(\sigma) \cos (2 \pi N \sigma)$ denotes the phase and $N$ is a free parameter of the formula called the corrugation number. The function $\alpha$ is given by

$$
\alpha(\sigma):=J_{0}^{-1}\left(\frac{\left\|\left(f_{0} \circ \gamma\right)^{\prime}(\sigma)\right\|}{r(\sigma)}\right)
$$

where $J_{0}(x)=\int_{0}^{1} \cos (x \cos 2 \pi u) \mathrm{d} u$ is the Bessel function of order zero, $J_{0}^{-1}$ is the inverse of the restriction of $J_{0}$ to the interval $[0, z]$, and $z \approx 2.4$ is the smallest positive root of $J_{0}$. Since $J_{0} \leqslant 1$ the argument of the right member of (4.9) must be smaller or equal to 1 . In other words, the shortness condition $\left\|\left(f_{0} \circ \gamma\right)^{\prime}(s)\right\| \leqslant r(s)$ is imposed by the Convex Integration Formula. The corrugated curve $\Gamma$ is the integral of a vector that oscillates $N$ times along $f_{0} \circ \gamma$. Because the norm of this vector is $r(s)$ the curve $\Gamma$ has the required target speed. The particular choice for $\alpha$ ensures that $\Gamma$ is close to $f_{0} \circ \gamma$ as illustrated on Figure 3. See [5] for more details.
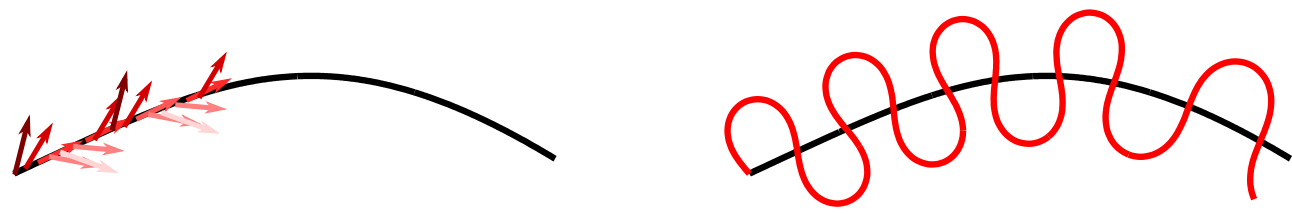

Figure 3. Left: the vector $r(\cos \theta \mathbf{t}(u)+\sin \theta \mathbf{n}(u))$ attached at each point of $f_{0} \circ \gamma$ oscillates $N$ times. Right: the resulting corrugated curve $\Gamma$.

Boundary condition. Our approach consists in applying the Convex Integration Formula (4.8) to a family of curves $\gamma_{p}:[0,1] \rightarrow \widetilde{\mathcal{D}}$ that foliates the domain $\widetilde{\mathcal{D}}$ and such that $p=\gamma_{p}(0)$ runs through the south boundary of $\widetilde{\mathcal{D}}$ while $\gamma_{p}(1)$ lies on the north boundary. However, this construction would create corrugations parallel to the boundaries of the domain (see Equation 5.16 and Lemma 4) and the isometric error would not be reduced in the horizontal direction (see Lemma 5). To overcome this difficulty, we propose below to shift the phase in Formula (4.8) by translating the parameter domain of each curve $\gamma_{p}$.

Convex Integration with phase shift. It is convenient to define the family of curves $\left(\gamma_{p}\right)_{p}$ as the flow lines of a (lift of a) smooth vector field $w$ over $\mathcal{D}$. We suppose here that this vector field is nowhere horizontal, in other words $w=X e_{X}+Y e_{Y}$ with $Y \neq 0$, say $Y>0$. Denoting by $\tilde{w}$ its lift over $\widetilde{\mathcal{D}}$ it follows that for every point $p=\left(x,-y_{\infty}\right)$ on the south boundary of $\widetilde{\mathcal{D}}$ the flow line of $\tilde{w}$ starts at $p$ and joins the north boundary of $\widetilde{\mathcal{D}}$. Let $\gamma_{p}:\left[a_{p}, b_{p}\right] \rightarrow \widetilde{\mathcal{D}}$ be this flow line with the initial condition $\gamma_{p}\left(a_{p}\right)=p$. When $p$ runs through the south boundary, the family $\left(\gamma_{p}\right)_{p}$ of flow lines foliates $\widetilde{\mathcal{D}}$ and every $\gamma_{p}$ cuts each latitude $\{y=$ const $\}$ exactly once. We apply the Convex Integration Formula (4.8) to the one-parameter family of curves $\left(\gamma_{p}\right)_{p}$ and to a target speed function $r: \widetilde{\mathcal{D}} \rightarrow \mathbb{R}_{>0}$ satisfying the shortness condition $\left\|\left(w \cdot f_{0}\right)([q])\right\| \leqslant r(q)$ for all $q \in \widetilde{\mathcal{D}}$, and descending to the quotient over $\mathcal{D}$. Since the isometric error cancels along the south and north boundaries of $\mathcal{D}$ we also impose the equality $\left\|\left(w \cdot f_{0}\right)([p])\right\|=r(p)$ along 
those boundaries. We thus produce a new map $\widetilde{F}: \widetilde{\mathcal{D}} \rightarrow \mathbb{R}^{3}$ :

$$
\widetilde{F} \circ \gamma_{p}(s):=f_{0}([p])+\int_{a_{p}}^{s} r\left(\gamma_{p}(\sigma)\right)\left(\cos \theta \mathbf{t}\left(\gamma_{p}(\sigma)\right)+\sin \theta \mathbf{n}\left(\gamma_{p}(\sigma)\right)\right) \mathrm{d} \sigma,
$$

where $[p]$ is the canonical projection of $p$ in $\mathcal{D}$ and $\theta:=\alpha(\sigma) \cos (2 \pi N \sigma)$. In this last formula, the corrugation number $N$ is independent of $p$ and

$$
\alpha(\sigma):=J_{0}^{-1}\left(\frac{\left\|\left(f_{0} \circ \gamma_{p}\right)^{\prime}(\sigma)\right\|}{r\left(\gamma_{p}(\sigma)\right)}\right) .
$$

See Figure 4. Given the vector field $w$, we emphasize that the lower bound $a_{p}$ in the domain of
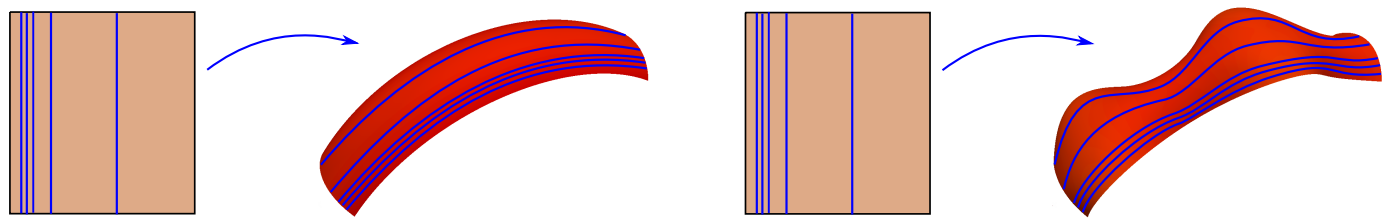

FIGURE 4. Left, each curve in $\widetilde{\mathcal{D}}$ is sent by the initial embedding $f_{0}$ to a short curve in $\mathbb{R}^{3}$ (only a portion is represented). Right, we apply the one dimensional convex integration to each curve in the family to obtain a corrugated surface $\widetilde{F}(\widetilde{\mathcal{D}})$.

definition $\left[a_{p}, b_{p}\right]$ of the flow line $\gamma_{p}$ can be chosen arbitrarily. In particular, when $p \mapsto a_{p}$ is $C^{\infty}$ then so is the map $(x, s) \mapsto \gamma(x, s)=\gamma_{\left(x,-y_{\infty}\right)}(s)$. In order to have an appropriate control over the transversal derivatives of $\widetilde{F}$ it appears crucial to impose a constant direction to the partial derivative $\frac{\partial \gamma}{\partial x}$. This can only be achieved by choosing $p \rightarrow a_{p}$ to be affine. See Lemmas 4 and 5 and their proof.

Lemma 2. Suppose that $a_{p}$ is an affine function of $p$ and let $\Delta=a_{p+2 \pi e_{X}}-a_{p}$. If $N \Delta \in \mathbb{Z}$, then $\widetilde{F}$ descend to the quotient on $\mathcal{D}$.

Proof. Let $q=\gamma_{p}(s)$. Note that $q+2 \pi e_{X}=\gamma_{p+2 \pi e_{X}}(s+\Delta)$ since $\widetilde{w}$ is invariant by a translation of vector $2 \pi e_{X}$ and $a_{p+2 \pi e_{X}}=a_{p}+\Delta$. For short, we write $\mathbf{e}^{i \theta}(p, \sigma)$ for $\cos \theta \mathbf{t}\left(\gamma_{p}(\sigma)\right)+$ $\sin \theta \mathbf{n}\left(\gamma_{p}(\sigma)\right)$. We thus have

$$
\widetilde{F}\left(q+2 \pi e_{X}\right)=f_{0}\left(\left[p+2 \pi e_{X}\right]\right)+\int_{a_{p+2 \pi e_{X}}}^{s+\Delta} r\left(\gamma_{p+2 \pi e_{X}}(\sigma)\right) \mathbf{e}^{i \theta}\left(p+2 \pi e_{X}, \sigma\right) \mathrm{d} \sigma
$$

The change of variables $u=\sigma-\Delta$ gives

$$
\widetilde{F}\left(q+2 \pi e_{X}\right)=f_{0}([p])+\int_{a_{p}}^{s} r\left(\gamma_{p+2 \pi e_{X}}(u+\Delta)\right) \mathbf{e}^{i \theta}\left(p+2 \pi e_{X}, u+\Delta\right) \mathrm{d} u
$$

On the one hand, $\gamma_{p+2 \pi e_{X}}(u+\Delta)=\gamma_{p}(u)+2 \pi e_{X}$ so that $r\left(\gamma_{p+2 \pi e_{X}}(u+\Delta)\right)=r\left(\gamma_{p}(u)\right)$, $\mathbf{t}\left(\gamma_{p+2 \pi e_{X}}(u+\Delta)\right)=\mathbf{t}\left(\gamma_{p}(u)\right)$ and $\mathbf{n}\left(\gamma_{p+2 \pi e_{X}}(u+\Delta)\right)=\mathbf{n}\left(\gamma_{p}(u)\right)$. On the other hand,

$$
\begin{aligned}
\theta\left(p+2 \pi e_{X}, u+\Delta\right) & =\alpha_{p+2 \pi e_{X}}(u+\Delta) \cos (2 \pi N(u+\Delta)) \\
& =\alpha_{p}(u) \cos (2 \pi N u+2 \pi N \Delta) .
\end{aligned}
$$

Hence, if $N \Delta$ is an integer, we conclude that $\widetilde{F}\left(q+2 \pi e_{X}\right)=\widetilde{F}(q)$. 
AN EXPLICIT ISOMETRIC REDUCTION OF THE UNIT SPHERE INTO AN ARBITRARILY SMALL BALLDEDICATED TO THE MEMO

Under the hypothesis in the lemma, we obtain a quotient map $F: \mathcal{D} \rightarrow \mathbb{R}^{3}$ whose effect is to corrugate $R_{0}$ in the direction $w$ so that each curve $s \mapsto F \circ\left[\gamma_{p}(s)\right]$ has the desired speed. In other words, $\gamma_{p}^{\prime}(s)^{t} I_{F} \gamma_{p}^{\prime}(s)$ and $r^{2}\left(\gamma_{p}(s)\right)$ coincide. An important feature of convex integration is that we can enforce $F$ to be as close as desired to $f_{0}$. In fact we have the following $C^{0}$ density Formula, see [5, Lemma 1],

$$
\left\|F-f_{0}\right\|_{\infty}=O\left(\frac{1}{N}\right) .
$$

We cannot expect such a closeness formula for the differentials $d F$ and $d f_{0}$. However, the vector field $\nu=\frac{\partial \gamma}{\partial x}$ descends to the quotient and gives a vector field, still denoted by $\nu$, along which an analogous formula holds true.

Lemma 3. We have

$$
\left\|\nu \cdot F-\nu \cdot f_{0}\right\|_{\infty}=O\left(\frac{1}{N}\right)
$$

where $\nu \cdot F$ is the derivative $d F(\nu)$ of $F$ in direction $\nu$.

Proof. Let $\kappa(x, \sigma) \mathrm{d} \sigma$ be the integrant in Formula (4.10). Recalling that $p=\left(x,-y_{\infty}\right)=$ $\gamma\left(x, a_{p}\right)$, we write

$$
\tilde{F} \circ \gamma(x, s)=f_{0}([p])+\int_{a_{p}}^{s} \kappa(x, \sigma) \mathrm{d} \sigma=f_{0}\left(\left[\gamma\left(x, a_{p}\right)\right]\right)+\int_{a_{p}}^{s} \kappa(x, \sigma) \mathrm{d} \sigma
$$

We have $(\nu \cdot \tilde{F})(\gamma(x, s))=\frac{\partial}{\partial x}(\tilde{F} \circ \gamma(x, s))$, hence

$$
(\nu \cdot \tilde{F})(\gamma(x, s))=A(x, s)+B(x) \text {, }
$$

where

and

$$
A(x, s):=\left(\nu \cdot f_{0}\right)\left(\left[\gamma\left(x, a_{p}\right)\right]\right)+\int_{a_{p}}^{s} \frac{\partial}{\partial x} \kappa(x, \sigma) \mathrm{d} \sigma
$$

$$
B(x):=\frac{\partial a_{p}}{\partial x}\left(\left(w \cdot f_{0}\right)\left(\left[\gamma\left(x, a_{p}\right)\right]\right)-\kappa\left(x, a_{p}\right)\right) .
$$

We observe that for every $x, A(x, s)$ is a Convex Integration formula similar to (4.8) applied to $\nu \cdot f_{0}$ instead of $f_{0}$. By the $C^{0}$ density Formula (4.12) we obtain

$$
\left\|A(x, s)-\left(\nu \cdot f_{0}\right)([\gamma(x, s)])\right\|_{\infty}=O\left(\frac{1}{N}\right) .
$$

Since $r(p)=\left\|\left(w \cdot f_{0}\right)([p])\right\|$ for every $p=\gamma\left(x, a_{p}\right)$ along the south boundary of $\widetilde{\mathcal{D}}$, it results from (4.11) that $\kappa\left(x, a_{p}\right)=\left(w \cdot f_{0}\right)\left(\left[\gamma\left(x, a_{p}\right)\right]\right)$. We conclude that $B(x)=0$.

\section{Reducing the PRimitive COORdinates}

In order to reduce the isometric error $E\left(f_{0}\right)$ we shall build three maps $f_{i}: \mathcal{D} \rightarrow \mathbb{R}^{3}, i=1,2,3$, so as to reduce the three primitive coordinates of $I_{h}-I_{f_{0}}$, see Equation (2.2). Each map $f_{i}$ is constructed in two steps. The first step builds a map $F_{i}$ from $f_{i-1}$ by applying our Convex Integration Formula (4.10). Because this map does not connect the two polar caps, we interpolate between $F_{i}$ and $f_{i-1}$ in a second step to get a smooth map $f_{i}$. We call the applications of these two steps a CI process. In this section we only consider the first step of the CI process and show that the $i$ th primitive coordinate of $I_{h}-I_{F_{i}}$ can be made arbitrarily small, see Lemma 5 .

The family $\left(\gamma_{p}\right)_{p}$ of curves is given by the flow curves of a vector field $w_{i}$ to be defined below and the target speed by:

$$
r=\sqrt{\left\|w_{i} \cdot f_{i-1}\right\|^{2}+\rho_{i}}
$$


where $\rho_{i}=\rho_{i}\left(I_{h}-I_{f_{i-1}}\right)$ is the $i$ th primitive coordinate of $I_{h}-I_{f_{i-1}}$. Note that the shortness condition implies that $\rho_{i}$ must be non-negative. We introduce for $i=1,2,3$, the intermediate metrics

$$
\mu_{i}:=I_{f_{i-1}}+\rho_{i}\left(I_{h}-I_{f_{i-1}}\right) \ell_{i} \otimes \ell_{i}=\rho_{i}\left(I_{h}\right) \ell_{i} \otimes \ell_{i}+\sum_{j \in\{1,2,3\}, j \neq i} \rho_{j}\left(I_{f_{i-1}}\right) \ell_{j} \otimes \ell_{j},
$$

and the constant vector fields $v_{i}$ in the direction of ker $\ell_{i}$ :

$$
v_{1}=\frac{1}{\sqrt{2}}\left(\begin{array}{c}
-1 \\
1
\end{array}\right), \quad v_{2}=-\frac{1}{\sqrt{2}}\left(\begin{array}{l}
1 \\
1
\end{array}\right), \quad v_{3}=\left(\begin{array}{c}
-1 \\
0
\end{array}\right)
$$

We define $w_{i}$ as the vector field $\mu_{i}$-orthogonal to $v_{i}$ with the normalization $\ell_{i}\left(w_{i}\right)=1$. Our goal is to construct $F_{i}$ such that $\mu_{i}=I_{F_{i}}$ up to $O\left(1 / N_{i}\right)$. Because $v_{i} \in \operatorname{ker} \ell_{i}$, we have $\mu_{i}\left(v_{i},.\right)=I_{f_{i-1}}\left(v_{i},.\right)$. It ensues that $v_{i} \cdot F_{i}$ should be $v_{i} \cdot f_{i-1}$ up to $O\left(1 / N_{i}\right)$. We achieve this condition thanks to Lemma 3 by choosing the map $p \mapsto a_{p}$ so that $\nu=\partial \gamma / \partial x$ is proportional to $v_{i}$. This choice actually fixes $a_{p}$ :

Lemma 4. $\nu \in \operatorname{ker} \ell_{i} \Longleftrightarrow a_{p}=\ell_{i}(p-(0,0))+$ const.

In particular, this eliminates the naive choice $a_{p}=0$, or more generally $a_{p}=$ const, for $i=1$ or 2 . Here, we use the notation $p-(0,0)$ for the vector with the same coordinates as point $p$.

Proof. Let $\tilde{w}_{i}$ be the lift of $w_{i}$ in $\widetilde{\mathcal{D}}$. From $\frac{\partial \gamma_{p}}{\partial s}=\tilde{w}_{i}\left(\gamma_{p}\right)$ and $\ell_{i}\left(\tilde{w}_{i}\right)=1$ we obtain $\ell_{i}\left(\frac{\partial \gamma_{p}}{\partial s}\right)=1$ and by integrating between $a_{p}$ and $s$ :

$$
\ell_{i}\left(\gamma_{p}(s)-p\right)=s-a_{p}
$$

since $\gamma_{p}\left(a_{p}\right)=p$. Differentiating with respect to $x$, the above relation leads to

$$
\ell_{i}\left(\frac{\partial \gamma_{p}}{\partial x}-e_{X}\right)=-\frac{\partial a_{p}}{\partial x}
$$

Hence, $\frac{\partial \gamma_{p}}{\partial x} \in \operatorname{ker} \ell_{i}$ is equivalent to $\frac{\partial a_{p}}{\partial x}=\ell_{i}\left(e_{X}\right)$. We conclude by integrating this last relation, recalling that $p-(0,0)=x e_{X}-y_{\infty} e_{Y}$.

Taking const $=0$ in Lemma 4 , we fix the parametrization of each $\gamma_{p}$ by the initial condition:

$$
\gamma_{p}\left(a_{p}\right)=p \quad \text { with } \quad a_{p}:=\ell_{i}(p-(0,0)) .
$$

Note that $\ell_{i}(p-(0,0))$ is the algebraic distance of $p$ to the line through $(0,0)$ in the direction $v_{i}$. This way, for any straight line parallel to $\operatorname{ker} \ell_{i}$ all the flow curves cut this line with the same parameter $s$. More precisely, we have

$$
s=\ell_{i}\left(\gamma_{p}(s)-(0,0)\right) .
$$

Looking at (4.10), we call the set of points $\gamma_{p}(s)$ for which the phase $\theta$ cancels the ridge lines of our corrugated map $F_{i}$. Equation (5.16) tells us that the ridge lines are straight lines parallel to $v_{i}$. See Figure 5.

We finally choose the corrugation number $N_{i}$ satisfying the hypothesis of Lemma 2.

Lemma 5. Suppose that $w_{i}$ is nowhere horizontal, then $\nu=\partial \gamma / \partial x$ does not vanish and is proportional to $v_{i}$. In particular, one has

$$
\left\|v_{i} \cdot F_{i}-v_{i} \cdot f_{i-1}\right\|_{\infty}=O\left(1 / N_{i}\right) .
$$

Proof of Lemma 5. Recall from Lemma 3 that $\left\|\nu \cdot F_{i}-\nu \cdot f_{i-1}\right\|_{\infty}=O\left(\frac{1}{N_{i}}\right)$ where $\nu=\frac{\partial \gamma}{\partial x}$. Let $\tilde{w}_{i}$ be the lift of $w_{i}$ in $\widetilde{\mathcal{D}}$ and let $\Phi$ be the flow of $\tilde{w}_{i}$. Recalling that $p=(0,0)+x e_{X}-y_{\infty} e_{Y}$, we have in particular $\Phi(p, 0)=p$ and $\Phi\left(p, s-a_{p}\right)=\gamma(x, s)$. Note that $\Phi_{s}(p):=\Phi(p, s)$ is a local diffeomorphism and by definition $\frac{\partial \Phi}{\partial s}=\tilde{w}_{i}$. By differentiating $s=\ell_{i}(\gamma(x, s)-(0,0))$ with 
AN EXPLICIT ISOMETRIC REDUCTION OF THE UNIT SPHERE INTO AN ARBITRARILY SMALL BALLDEDICATED TO THE MEMO
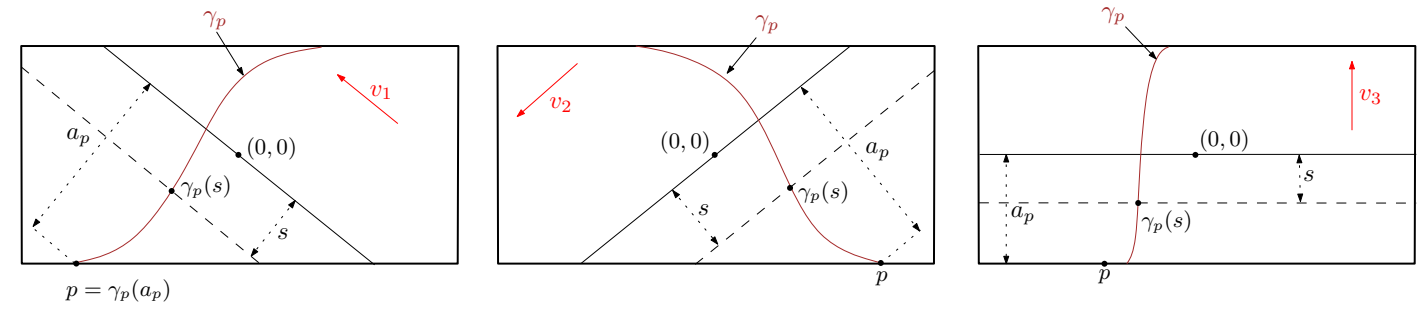

Figure 5. A curve $\gamma_{p}$ for each direction $v_{1}, v_{2}$ and $v_{3}$ of the ridge lines.

respect to $x$ we obtain $\ell_{i}\left(\frac{\partial \gamma}{\partial x}\right)=0$. In other words $\nu \propto v_{i}$. It thus remains to prove that $\nu$ does not cancel. On the one hand, we have

$$
\frac{\partial \gamma}{\partial x}=\frac{\partial}{\partial x} \Phi\left(p, s-a_{p}\right)=\mathrm{d} \Phi_{s-a_{p}} \cdot e_{X}-\frac{\partial a_{p}}{\partial x} \tilde{w}_{i}
$$

By differentiating $a_{p}=\ell_{i}(p-(0,0))$ with respect to $x$ we obtain $\frac{\partial a_{p}}{\partial x}=\ell_{i}\left(e_{X}\right)$. Whence,

$$
\frac{\partial \gamma}{\partial x}=\mathrm{d} \Phi_{s-a_{p}} \cdot e_{X}-\ell_{i}\left(e_{X}\right) \tilde{w}_{i}
$$

On the other hand, we trivially have $\mathrm{d} \Phi_{s-a_{p}} \cdot \tilde{w}_{i}=\tilde{w}_{i}$. By hypothesis, $e_{X}$ and $\tilde{w}_{i}$ are independent. Since $\Phi_{s}$ is a local diffeomorphism for every $s$, we infer that $\mathrm{d} \Phi_{s-a_{p}} \cdot e_{X}$ and $\mathrm{d} \Phi_{s-a_{p}} \cdot \tilde{w}_{i}$ are also independent. In turn, we conclude from (5.17) that $\frac{\partial \gamma}{\partial x} \neq 0$.

Lemma 6. Suppose that $w_{i}$ is nowhere horizontal, then

$$
\left\|\mu_{i}-I_{F_{i}}\right\|=O\left(1 / N_{i}\right) \quad \text { and } \quad \rho_{i}\left(I_{h}-I_{F_{i}}\right)=O\left(1 / N_{i}\right) .
$$

Proof. We first show that $\mu_{i}\left(u_{1}, u_{2}\right)-I_{F_{i}}\left(u_{1}, u_{2}\right)=O\left(1 / N_{i}\right)$ for $u_{1}, u_{2} \in\left\{v_{i}, w_{i}\right\}$. By definition $r^{2}=\mu_{i}\left(w_{i}, w_{i}\right)$, in other words $I_{F_{i}}\left(w_{i}, w_{i}\right)=\mu_{i}\left(w_{i}, w_{i}\right)$. We compute

$$
I_{F_{i}}\left(v_{i}, v_{i}\right)-\mu_{i}\left(v_{i}, v_{i}\right)=I_{F_{i}}\left(v_{i}, v_{i}\right)-I_{f_{i-1}}\left(v_{i}, v_{i}\right)=\left\langle v_{i} \cdot F_{i}-v_{i} \cdot f_{i-1}, v_{i} \cdot F_{i}+v_{i} \cdot f_{i-1}\right\rangle
$$

which is $O\left(1 / N_{i}\right)$ by Lemma 5 . Since $w_{i}$ and $v_{i}$ are $\mu_{i}$-orthogonal, we have

$$
I_{F_{i}}\left(w_{i}, v_{i}\right)-\mu_{i}\left(w_{i}, v_{i}\right)=I_{F_{i}}\left(w_{i}, v_{i}\right)=\left\langle w_{i} \cdot F_{i}, v_{i} \cdot F_{i}\right\rangle
$$

By differentiating (4.10) with respect to $s$ we get that $w_{i} \cdot F_{i}$ is a linear combination of $w_{i} \cdot f_{i-1}$ and the normal to the surface $f_{i-1}$. Using that $\left\langle w_{i} \cdot f_{i-1}, v_{i} \cdot f_{i-1}\right\rangle=\mu_{i}\left(w_{i}, v_{i}\right)=0$ it follows that $\left\langle w_{i} \cdot F_{i}, v_{i} \cdot f_{i-1}\right\rangle=0$. We can thus write

$$
I_{F_{i}}\left(w_{i}, v_{i}\right)-\mu_{i}\left(w_{i}, v_{i}\right)=\left\langle w_{i} \cdot F_{i}, v_{i} \cdot F_{i}-v_{i} \cdot f_{i-1}\right\rangle=O\left(1 / N_{i}\right)
$$

We conclude that $\left\|\mu_{i}-I_{F_{i}}\right\|=O\left(1 / N_{i}\right)$. We can now write,

$$
\rho_{i}\left(I_{h}-I_{F_{i}}\right)=\rho_{i}\left(I_{h}-\mu_{i}\right)+O\left(1 / N_{i}\right)
$$

By considering the $i$ th primitive coordinate of each member in (5.14) we obtain $\rho_{i}\left(\mu_{i}\right)=\rho_{i}\left(I_{h}\right)$, whence the desired result: $\rho_{i}\left(I_{h}-I_{F_{i}}\right)=O\left(1 / N_{i}\right)$.

\section{ITERATIVE CONSTRUCTION}

The effect of convex integration is to replace $f_{i-1}$ by a map $F_{i}$ whose first fundamental form is close to $\mu_{i}$. Considering the first step in the CI process, we note that the first primitive coordinate of $I_{h}-I_{F_{1}}$ cancels. However, the second step of the CI process consists in interpolating $F_{1}$ and $f_{0}$ to build $f_{1}$. This interpolation perturbs the primitive coordinates of $I_{h}-I_{f_{1}}$ and in particular its first coordinate may be negative. A similar phenomenon holds for the two subsequent CI processes. In the end, the loop condition (C4) may not be satisfied by the pair 
$\left(f_{3}, h\right)$. This would prevent us from applying other CI processes to further reduce the isometric error. To overcome this difficulty we replace the target metric $I_{h}$ by an increasing sequence of linearly interpolated metrics

$$
I_{k}:=\tau_{k}^{2} I_{f_{0}}+\left(1-\tau_{k}^{2}\right) I_{h}
$$

that converges toward $I_{h}$, where $\left(\tau_{k}\right)_{k \geqslant 0}$ is a strictly decreasing sequence of numbers converging to 0 with $\tau_{0}=1$. Note that $I_{f_{0}}<I_{k}<I_{h}$ over $\mathcal{D}$. After three convex integrations with respect to $I_{1}$, the difference $I_{1}-I_{f_{3}}$ may have negative primitive coordinates, but by choosing sufficiently large corrugation numbers, this difference will be small enough so that $I_{2}-I_{f_{3}}$ will have positive primitive coordinates away from the boundary $\partial \mathcal{D}$ of $\mathcal{D}$. This would allows us to iterate the process, reducing the isometric error with respect to $I_{2}, I_{3}, \ldots$ until it vanishes in the limit. Nevertheless, in the present construction there is yet another issue: because $I_{h}=I_{f_{0}}$ along $\partial \mathcal{D}$ we must have $I_{1}=I_{h}$ along this boundary and it may happen again that the second and third primitive coordinates of $I_{1}-I_{f_{1}}$ are negative in the vicinity of $\partial \mathcal{D}$. This would prevent us from building $f_{2}$ over the entire domain $\mathcal{D}$. We bypass this second difficulty by considering a nested sequence of equatorial sub-ribbons of $\mathcal{D}$

$$
\mathcal{D}_{0,3} \subset \mathcal{D}_{1,1} \subset \mathcal{D}_{1,2} \subset \mathcal{D}_{1,3} \quad \subset \quad \ldots \quad \subset \quad \mathcal{D}_{k, 1} \subset \mathcal{D}_{k, 2} \subset \mathcal{D}_{k, 3} \quad \subset \quad \ldots \quad \ldots \quad \mathcal{D}
$$

of the form $\left.\mathcal{D}_{k, i}:=\mathbb{S}^{1} \times\right]-y_{k, i}, y_{k, i}\left[\right.$ where $y_{0,3}<y_{1,1}<y_{1,2}<y_{1,3}<y_{2,1} \ldots$ are chosen such that $\left\|I_{h}-I_{f_{0}}\right\| \leqslant \tau_{k}^{2}$ on $\mathcal{D} \backslash \mathcal{D}_{k, 3}$ (this is possible since $I_{h}-I_{f_{0}}=0$ on $\partial \mathcal{D}$ and by continuity). In particular, $\left(y_{k, 3}\right)_{k}$ converges to $y_{\infty}$. We next replace each $I_{k}$ by three altered metrics $I_{k, 1}, I_{k, 2}, I_{k, 3}$ defined by

$$
I_{k, i}(x, y):=\left(1-\lambda_{k, i}(|y|)\right) I_{f_{0}}(x, y)+\lambda_{k, i}(|y|) I_{k}(x, y)
$$

where $0 \leqslant \lambda_{k, i} \leqslant 1$ is an interpolating function with support $\left[0, \frac{y_{k, i-1}+y_{k, i}}{2}\right]$ and such that $\lambda_{k, i}(y)=1$ if and only if $0 \leqslant y \leqslant y_{k, i-1}$. See Fig 6 . For future reference, we set $y_{k, i-\frac{1}{2}}=$
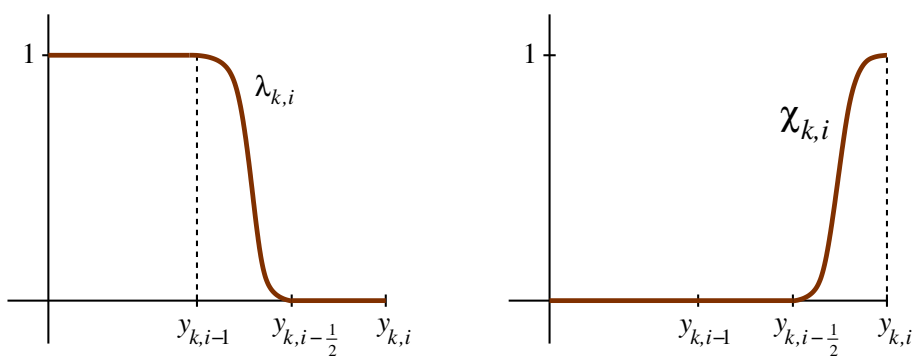

FIGURE 6. Left, the interpolating function $\lambda_{k, i}$ is defined over $\left[0, y_{k, i}\right]$. Right, the $C^{\infty}$ function $\chi_{k, i}$.

$\frac{y_{k, i-1}+y_{k, i}}{2}$. Remark that

- $I_{k, i}=I_{f_{0}}$ over $\mathcal{D} \backslash\left(\mathbb{S}^{1} \times\left[-y_{k, i-\frac{1}{2}}, y_{k, i-\frac{1}{2}}\right]\right)$,

- $I_{k, i}=I_{k}$ over $\mathcal{D}_{k, i-1}$,

- $I_{k, i}<I_{k, i+1}$ over $\left(\mathbb{S}^{1} \times\right]-y_{k, i+\frac{1}{2}}, y_{k, i+\frac{1}{2}}[) \backslash\left(\mathbb{S}^{1} \times\left[-y_{k, i-1}, y_{k, i-1}\right]\right)$.

See Figure 7.

The sequence of metrics $\left(I_{k, i}\right)_{k, i}$ is increasing. Formally, for $k<\ell$ or for $k=\ell$ and $i<j$, we have

$$
I_{f_{0}} \leqslant I_{k, i} \leqslant I_{\ell, j} \leqslant I_{h} \quad \text { over } \mathcal{D} \text {. }
$$

We now recursively build a sequence of maps $f_{k, i}$ converging towards an isometric map and satisfying $f_{k, i}=f_{0}$ over $\mathcal{D} \backslash \mathcal{D}_{k, i}$, for $i=1,2,3$ and $k \geqslant 1$. Here and below we use the usual 
AN EXPLICIT ISOMETRIC REDUCTION OF THE UNIT SPHERE INTO AN ARBITRARILY SMALL BALLDEDICATED TO THE MEMO

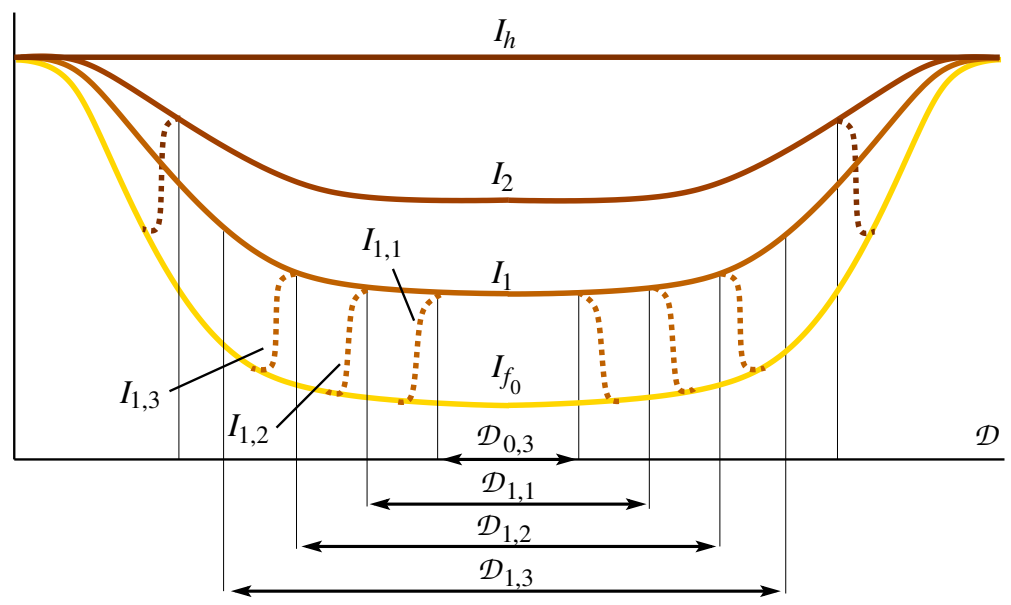

FiguRE 7. The nested domains and the interpolated metrics.

circular convention $*_{k, 0}=*_{k-1,3}$. We perform a convex integration process on the domain $\mathcal{D}_{k, i}$ from an embedding $f_{k, i-1}$ with the target metric $\mu_{k, i}$ (to be defined below) derived from $I_{k, i}$ in the direction $w_{k, i}$. In Lemma 12 at the end of Section 8 we prove that $w_{k, i}$ is never horizontal. As for $\mathcal{D}$, the boundary of $\mathcal{D}_{k, i}$ is composed of two latitudes, one in each hemisphere. Given a point $p=\left(x,-y_{k, i}\right)$ on the south boundary of $\mathcal{D}_{k, i}$, we consider the flow line of $w_{k, i}$ starting at $p$. This flow line is never horizontal and crosses the domain to reach its north boundary. We denote by $\gamma_{p}:\left[a_{p}, b_{p}\right] \rightarrow \widetilde{\mathcal{D}}_{k, i}$ the flow line of the lift $\widetilde{w}_{k, i}$ of $w_{k, i}$ with the initial condition

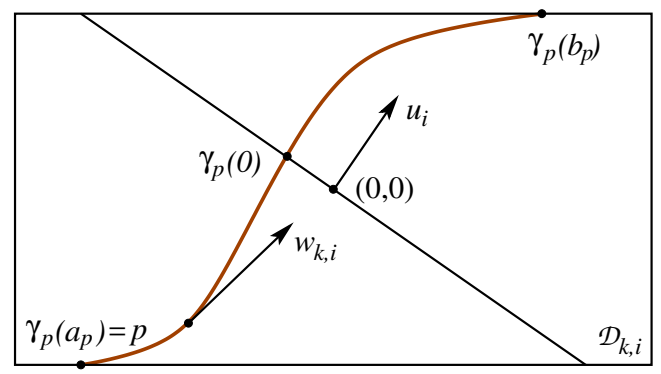

Figure 8. A flow line of $w_{k, i}$ in the parametric domain $\mathcal{D}_{k, i}$.

$\gamma_{p}\left(a_{p}\right)=p$, where $a_{p}=\ell_{i}(p-(0,0))$. Here, $b_{p}$ is such that $\gamma_{p}\left(b_{p}\right)$ belongs to the north boundary of $\widetilde{\mathcal{D}}_{k, i}$. See Fig 8. Replacing the curve $\gamma$ of Formula (4.8) by $\gamma_{p}$ leads to our formula for the corrugated map $\widetilde{F}_{k, i}: \widetilde{\mathcal{D}}_{k, i} \rightarrow \mathbb{R}^{3}$ :

$$
\widetilde{F}_{k, i} \circ \gamma_{p}(s):=f_{k, i-1}([p])+\int_{a_{p}}^{s} r\left(\gamma_{p}(\sigma)\right)\left(\cos \theta \mathbf{t}\left(\gamma_{p}(\sigma)\right)+\sin \theta \mathbf{n}\left(\gamma_{p}(\sigma)\right)\right) \mathrm{d} \sigma
$$

where $\mathbf{t}\left(\gamma_{p}(\sigma)\right)$ is the unit vector tangent to the curve $f_{k, i-1} \circ\left[\gamma_{p}\right]$ and $\mathbf{n}\left(\gamma_{p}(\sigma)\right)$ is the normal to the surface $f_{k, i-1}\left(\mathcal{D}_{k, i}\right)$ at the point $f_{k, i-1}\left(\left[\gamma_{p}(\sigma)\right]\right)$. Here, $\theta:=\theta(p, \sigma)=\alpha_{p}(\sigma) \cos \left(2 \pi N_{k, i} \sigma\right)$ with

$$
\alpha_{p}(\sigma):=J_{0}^{-1}\left(\frac{\left\|\left(f_{k, i-1} \circ\left[\gamma_{p}\right]\right)^{\prime}(\sigma)\right\|}{r\left(\gamma_{p}(\sigma)\right)}\right)
$$

and $r\left(\gamma_{p}(\sigma)\right)$ is the target speed $\sqrt{\mu_{k, i}\left(w_{k, i}, w_{k, i}\right)}$ at the point $\left[\gamma_{p}(\sigma)\right]$. 
LEMMA 7. If $2 \pi N_{k, i} \ell_{i}\left(e_{X}\right) \in \mathbb{Z}$, then $\widetilde{F}_{k, i}$ descend to the quotient on $\mathcal{D}_{k, i}$.

Proof. The lemma follows from Lemma 2, noting that $a_{p}=\ell_{i}(p-(0,0))$ implies $a_{p+2 \pi e_{X}}=$ $a_{p}+2 \pi \ell_{i}\left(e_{X}\right)$.

From now on we choose $N_{k, i}$ so that the condition in Lemma 7 is fulfilled and we denote by $F_{k, i}: \mathcal{D}_{k, i} \rightarrow \mathbb{R}^{3}$ the resulting quotient map. Since the resulting map $F_{k, i}$ is only defined over $\mathcal{D}_{k, i}$ we then perform the second step of the CI process by defining $f_{k, i}$ with the following interpolation formula:

$$
f_{k, i}(x, y):=\left(1-\chi_{k, i}(|y|)\right) F_{k, i}(x, y)+\chi_{k, i}(|y|) f_{k, i-1}(x, y) .
$$

where $0 \leqslant \chi_{k, i} \leqslant 1$ is a plateau function with support $] y_{k, i-\frac{1}{2}}, y_{k, \infty}[$ satisfying

$$
\left.0<\chi_{k, i}(y)<1 \Longrightarrow y \in\right] y_{k, i-\frac{1}{2}}, y_{k, i}[
$$

see Figure 6. We point out that the restriction of $f_{k, i}$ over $\mathcal{D} \backslash \mathcal{D}_{k, i}$ is equal to $f_{0}$ and that $f_{k, i}=F_{k, i}$ in $\mathcal{D}_{k, i-1}$. In particular, $f_{k, i}$ connects in a $C^{1}$ manner to the north and south caps along $\partial \mathcal{D}$.

\section{The Stage Theorem and the Convergence towards a Reduced Sphere}

In this section and the following one we state and prove the convergence of the sequence $\left(f_{k, 3}\right)_{k}$ towards an isometric map.

LEMma 8. We have $\left\|f_{k, i}-F_{k, i}\right\|_{C^{1}}=O\left(1 / N_{k, i}\right)$. In particular, $\left\|I_{f_{k, i}}-I_{F_{k, i}}\right\|=O\left(1 / N_{k, i}\right)$.

Proof. We detail the proof for $k=1$. The general case follows easily. We have $f_{1, i}-F_{1, i}=$ $\chi_{1, i}\left(f_{1, i-1}-F_{1, i}\right)$. From (4.12) we directly obtain $\left\|f_{1, i-1}-F_{1, i}\right\|_{C^{0}}=O\left(1 / N_{1, i}\right)$. For any vector field $U$ we have

$$
\begin{aligned}
U \cdot f_{1, i}-U \cdot F_{1, i} & =\left(U \cdot \chi_{1, i}\right)\left(f_{1, i-1}-F_{1, i}\right)+\chi_{1, i}\left(U \cdot f_{1, i-1}-U \cdot F_{1, i}\right) \\
& =O\left(1 / N_{1, i}\right)+\chi_{1, i}\left(U \cdot f_{1, i-1}-U \cdot F_{1, i}\right)
\end{aligned}
$$

When $U=v_{i}$ the second term in the right hand side is a $O\left(1 / N_{1, i}\right)$ by Lemmas 5 and 12 . When $U=w_{1, i}$ this second term vanishes since we either have $\chi_{1, i}=0$ or $w_{1, i} \cdot f_{1, i-1}=w_{1, i} \cdot F_{1, i}$. The latter equality can be seen by noting that $\chi_{1, i} \neq 0$ implies that the target metric $I_{1, i}$ is equal to $I_{f_{0}}$. Hence, the target speed in Formula (5.13) is equal to the initial speed $\left\|w_{1, i} \cdot f_{1, i-1}\right\|$ and consequently the restrictions of $F_{1, i}$ and $f_{1, i-1}$ over the flow lines of $w_{1, i}$ are translates of each other.

The introduction of the nested domains $\mathcal{D}_{1, i}$ and intermediate metrics $I_{1, i}$ allows us to control the additional error due to the interpolation step and provide the necessary slackness to iterate the CI process. Indeed, to compute $f_{1,1}$ we apply a CI process over $\mathcal{D}_{1,1}$ with the target metric

$$
\mu_{1,1}=\rho_{1}\left(I_{1,1}\right) \ell_{1} \otimes \ell_{1}+\rho_{2}\left(I_{f_{0}}\right) \ell_{2} \otimes \ell_{2}+\rho_{3}\left(I_{f_{0}}\right) \ell_{3} \otimes \ell_{3}=I_{f_{0}}+\rho_{1}\left(I_{1,1}-I_{f_{0}}\right) \ell_{1} \otimes \ell_{1}
$$

For the next CI process, we target the metric

$$
\mu_{1,2}=I_{f_{1,1}}+\rho_{2}\left(I_{1,2}-I_{f_{1,1}}\right) \ell_{2} \otimes \ell_{2}
$$

Hence, to process $F_{1,2}$ we need that $\rho_{2}\left(I_{1,2}\right)>\rho_{2}\left(I_{f_{1,1}}\right)$ over $\mathcal{D}_{1,2}$. We first show how to ensure this inequality over $\mathcal{D}_{1,2} \backslash \mathcal{D}_{1,1}$ by a convenient choice of convex integration and interpolation parameters. We have

$$
\rho_{2}\left(I_{1,2}\right)=\left(1-\lambda_{1,2}\right) \rho_{2}\left(I_{f_{0}}\right)+\lambda_{1,2} \rho_{2}\left(I_{1}\right) \quad \text { over } \quad \mathcal{D}_{1,2} \backslash \mathcal{D}_{1,1} .
$$


AN EXPLICIT ISOMETRIC REDUCTION OF THE UNIT SPHERE INTO AN ARBITRARILY SMALL BALLDEDICATED TO THE MEMO

Recalling that $I_{1}$ is a linear interpolation of $I_{f_{0}}$ and $I_{h}$ we also have that $\rho_{2}\left(I_{1}\right)>\rho_{2}\left(I_{f_{0}}\right)$. Since $I_{f_{1,1}}=I_{f_{0}}$ over $\mathcal{D}_{1,2} \backslash \mathcal{D}_{1,1}$, we deduce that $\rho_{2}\left(I_{1,2}\right)>\rho_{2}\left(I_{f_{1,1}}\right)$. We now check this inequality over $\mathcal{D}_{1,1}$. From Equation (7.21) and Lemma 6, one has

$$
\begin{aligned}
\rho_{2}\left(I_{f_{1,1}}\right) & =\rho_{2}\left(I_{f_{1,1}}-I_{F_{1,1}}\right)+\rho_{2}\left(\mu_{1,1}\right)+\rho_{2}\left(I_{F_{1,1}}-\mu_{1,1}\right) \\
& =\rho_{2}\left(I_{f_{1,1}}-I_{F_{1,1}}\right)+\rho_{2}\left(I_{f_{0}}\right)+O\left(1 / N_{1,1}\right) .
\end{aligned}
$$

Since $I_{1,2}=I_{1}$ over $\mathcal{D}_{1,1}$ and $\rho_{2}\left(I_{1}\right)>\rho_{2}\left(I_{f_{0}}\right)$ over $\mathcal{D}$, we get

$$
\rho_{2}\left(I_{1,2}\right)>\rho_{2}\left(I_{f_{0}}\right) \text { over } \mathcal{D}_{1,1} \cup \partial \mathcal{D}_{1,1} \text {. }
$$

By the interpolation formula, $f_{1,1}$ and $F_{1,1}$ are identical over $\mathcal{D}_{1,1} \backslash \mathcal{Z}_{1,1}$, where $\mathcal{Z}_{1,1}$ is the blending region of $F_{1,1}$ with $f_{0}$ :

$$
\mathcal{Z}_{1,1}:=\mathcal{D}_{1,1} \backslash\left(\mathbb{S}^{1} \times\left[-y_{1, \frac{1}{2}}, y_{1, \frac{1}{2}}\right]\right) .
$$

It follows from Equation (7.22) that $\rho_{2}\left(I_{f_{1,1}}\right)=\rho_{2}\left(I_{f_{0}}\right)+O\left(1 / N_{1,1}\right)$ over $\mathcal{D}_{1,1} \backslash \mathcal{Z}_{1,1}$. By $(7.23)$ we conclude that $\rho_{2}\left(I_{1,2}\right)>\rho_{2}\left(I_{f_{1,1}}\right)$ over the same domain. Over $\mathcal{Z}_{1,1}$, we have $I_{1,1}=I_{f_{0}}=\mu_{1,1}$, whence:

$$
\begin{aligned}
\rho_{2}\left(I_{f_{1,1}}\right) & =\rho_{2}\left(I_{f_{1,1}}-\mu_{1,1}\right)+\rho_{2}\left(\mu_{1,1}\right) \\
& =\rho_{2}\left(I_{f_{1,1}}-\mu_{1,1}\right)+\rho_{2}\left(I_{f_{0}}\right) .
\end{aligned}
$$

By Lemma 8, we have $I_{f_{1,1}}-I_{F_{1,1}}=O\left(1 / N_{1,1}\right)$. It follows from Lemma 6 that $I_{f_{1,1}}-\mu_{1,1}=$ $O\left(1 / N_{1,1}\right)$ and thus $\rho_{2}\left(I_{f_{1,1}}\right)$ can be made arbitrarily close to $\rho_{2}\left(I_{f_{0}}\right)$. By $(7.23)$ we conclude $\rho_{2}\left(I_{1,2}\right)>\rho_{2}\left(I_{f_{1,1}}\right)$ as desired.

By a convenient choice of $N_{1,1}$ we can thus iterate the process to build $f_{1,2}$. Similarly, a convenient choice of $N_{1,2}$ and $N_{1,3}$ allows to build $f_{1,3}$ and to ensure the loop condition for the pair $\left(f_{1,3}, I_{2}\right)$. More generally, recalling that $\left\|I_{h}-I_{f_{0}}\right\| \leqslant \tau_{k}^{2}$ on $\mathcal{D} \backslash \mathcal{D}_{k, 3}$, we can prove the following theorem (see Section 8):

Theorem 9 (Stage Theorem). Assume that for $i=1,2,3, \rho_{i}\left(I_{k, 1}\right)>\rho_{i}\left(f_{k-1,3}\right)$ over $\mathcal{D}_{k, 1}$. We can choose $N_{k, 1}, N_{k, 2}$ and $N_{k, 3}$ such that $f_{k, 3}$ satisfies

(1) for $i=1,2,3, \rho_{i}\left(I_{k+1,1}\right)>\rho_{i}\left(I_{f_{k, 3}}\right)$ over $\mathcal{D}_{k+1,1}$,

(2) $\left\|I_{k, 3}-I_{f_{k, 3}}\right\|_{\infty}^{1 / 2} \leqslant C \tau_{k-1}$,

(3) $\left\|d f_{k, 3}-d f_{k-1,3}\right\|_{\infty} \leqslant C^{\prime} \tau_{k-1}$,

where $C, C^{\prime}$ are constants independent of $k$.

Note that from (4.12) the difference $\left\|f_{k, 3}-f_{k-1,3}\right\|_{\infty}$ can be made arbitrarily small.

Corollary 10. If $\sum \tau_{k}<+\infty$ then any sequence $\left(f_{k, 3}\right)$ (glued with $f_{0}$ along $\left.\partial \mathcal{D}\right)$ satisfying condition (1-3) of the Stage Theorem $C^{1}$ converges towards an isometric map $f_{\infty}$ of $\mathbb{S}^{2}$. Furthermore for any $\varepsilon>0$ we can enforce that $\left\|f_{\infty}-f_{0}\right\|_{\infty}<\varepsilon$.

Proof. Because $\sum \tau_{k}<+\infty$ the sequence $\left(d f_{k, 3}\right)_{k}$ is Cauchy. The sequence $\left(f_{k, 3}\right)_{k}$ is obviously converging on $\partial \mathcal{D}$, hence is $C^{1}$ converging towards a $C^{1}$ map $f_{\infty}$. Now, by taking the limit on both sides of point (2) of the Stage Theorem we conclude that $f_{\infty}$ is an isometric map. Moreover, the control of the difference $\left\|f_{k, 3}-f_{k-1,3}\right\|_{\infty}$ in the Stage Theorem allows us to enforce $\left\|f_{\infty}-f_{0}\right\|_{\infty}<\varepsilon$.

Viewing $\mathbb{S}^{2}$ as the surface of the Earth, the map $f_{\infty}$ thus fits the Earth isometrically inside any ball containing $S_{0}=f_{0}\left(\mathbb{S}^{2}\right)$ in its interior, see Figure 9 . These rendered images were obtained from a discretization of $f_{1,3}$. The underlying computations were performed on a 16 core $\mathrm{CPU}$ with $64 \mathrm{~Gb}$ of RAM and took $2 \mathrm{~h} 46 \mathrm{mn}$. We used a regular grid of size 4,000 $\times 20,000$ to sample each embedding $f_{k, j}$. The following table gives the improvement of the metric at each of the three first steps. 

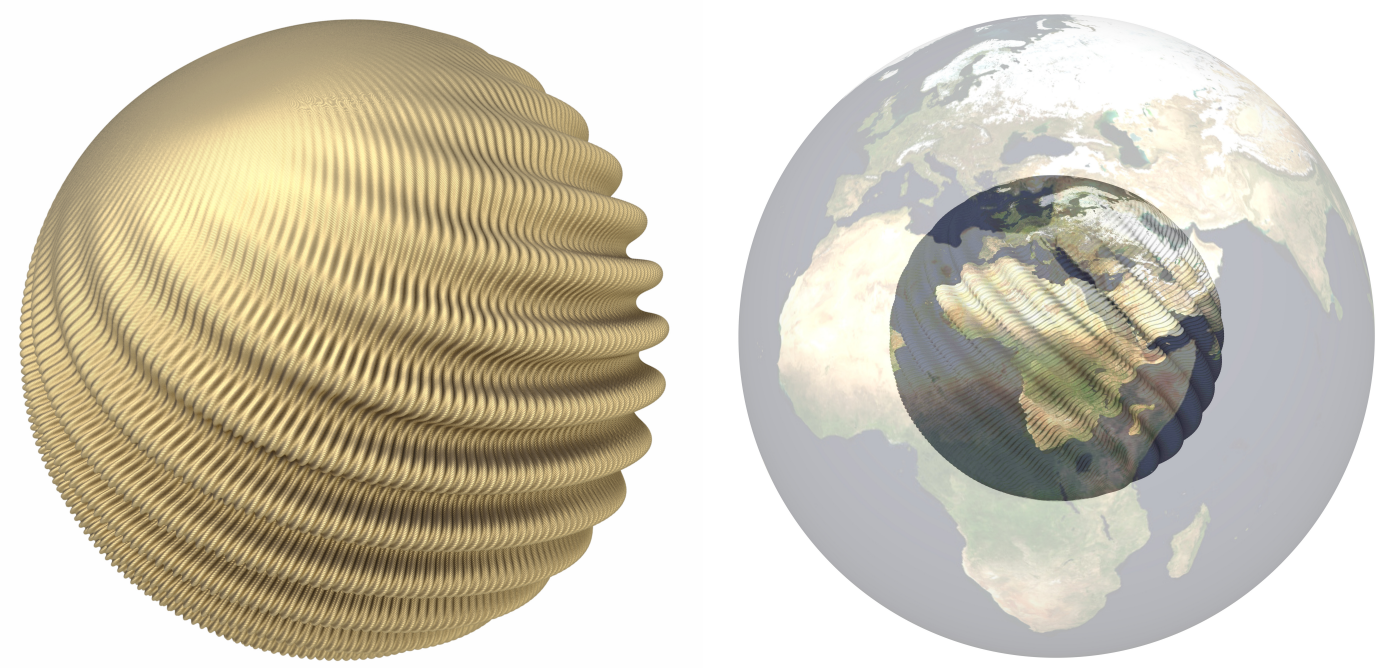

FiguRE 9. Left, an isometric sphere. Right, the reduced sphere is placed at the center of the original round sphere. Rendered images obtained after three steps and visualizing the image of $f_{1,3}$. Note that the difference between the image of $f_{1,3}$ and $f_{\infty}$ is imperceptible since the amplitude of the corrugations decrease dramatically.

\begin{tabular}{|c|c||c|c|c|c|}
\hline$k$ & $j$ & $N_{k, j}$ & $N_{k, j}^{*}$ & ave $(\sup )\left\|I_{h}-I_{f_{k, j}}\right\|$ & $\operatorname{ave}($ sup $)\left\|I_{k, j}-I_{f_{k, j}}\right\|$ \\
\hline 0 & - & - & - & $0.90(1.17)$ & - \\
\hline 1 & 1 & 4.72 & 21 & $0.83(1.03)$ & $0.14(0.24)$ \\
\hline 1 & 2 & 31.96 & 142 & $0.73(0.95)$ & $0.07(0.16)$ \\
\hline 1 & 3 & 334.92 & 997 & $0.66(0.94)$ & $0.03(0.18)$ \\
\hline
\end{tabular}

Note that the corrugation number $N_{k, j}$ is a real number but the number of visible ridge lines on the whole sphere is an integer denoted by $N_{k, j}^{*}$ in the table. The last two columns indicate the average (maximum) isometric error. For the last column, we observe that the isometric error is relative to the $I_{1, j}$ which are only equal over a subdomain (see Figure 7). The comparison of the maximum error is thus not very meaningful. The average value clearly decreases, showing the good approximation of the metric $I_{1}$ over $\mathcal{D}_{0,3}$.

\section{Proof of the Stage Theorem}

We write $f_{k, i}=I C\left(f_{k, i-1}, \mathcal{D}_{k, i}, N_{k, i}\right)$ to emphasize the dependency of the process on $\mathcal{D}_{k, i}$ and $N_{k, i}$. We set $D_{k, i}:=I_{k, i}-I_{f_{k, i-1}}$ and write

$$
D_{k, i}=\sum_{j=1}^{3} \rho_{j}\left(D_{k, i}\right) \ell_{j} \otimes \ell_{j}
$$

for its decomposition in the basis $\left(\ell_{1} \otimes \ell_{1}, \ell_{2} \otimes \ell_{2}, \ell_{3} \otimes \ell_{3}\right)$. We also define

$$
\mu_{k, i}:=I_{f_{k, i-1}}+\rho_{i}\left(D_{k, i}\right) \ell_{i} \otimes \ell_{i} .
$$

Theorem 11 (Step theorem). Assume that $\rho_{i}\left(D_{k, i}\right)>0$ over $\mathcal{D}_{k, i}$. We have

(1) $\left\|f_{k, i}-f_{k, i-1}\right\|_{\infty}=O\left(\frac{1}{N_{k, i}}\right)$

(2) $\left\|d f_{k, i}-d f_{k, i-1}\right\|_{\infty} \leqslant \frac{C_{k, i}}{N_{k, i}}+2 \sqrt{7}\left\|\rho_{i}\left(D_{k, i}\right)\right\|^{1 / 2}$ 
AN EXPLICIT ISOMETRIC REDUCTION OF THE UNIT SPHERE INTO AN ARBITRARILY SMALL BALLDEDICATED TO THE MEMO

(3) $\left\|\mu_{k, i}-I_{f_{k, i}}\right\|_{\infty} \leqslant \frac{C_{k, i}^{\prime}}{N_{k, i}}$

where $C_{k, i}$ and $C_{k, i}^{\prime}$ do not depend on $N_{k, i}$.

Proof. Point (1) is a direct consequence of (4.12) and (6.20). For Point (2) we have

$$
\left\|U \cdot f_{k, i}-U \cdot f_{k, i-1}\right\| \leqslant\left\|U \cdot f_{k, i}-U \cdot F_{k, i}\right\|+\left\|U \cdot F_{k, i}-U \cdot f_{k, i-1}\right\|
$$

for $U=w_{k, i}$ or $U=v_{i}$, and by Lemma 8:

$$
\left\|U \cdot f_{k, i}-U \cdot F_{k, i}\right\| \leqslant \frac{C_{1}}{N_{k, i}}
$$

where $C_{1}$ is a constant that depends on the parameters at the step $k, i$. On the other hand, we have by a direct application of Lemma 4 in [5]:

$$
\left\|w_{k, i} \cdot F_{k, i}-w_{k, i} \cdot f_{k, i-1}\right\| \leqslant \sqrt{7}\left\|\rho_{i}\left(D_{k, i}\right)\right\|^{1 / 2} .
$$

We also have

$$
\left\|v_{i} \cdot F_{k, i}-v_{i} \cdot f_{k, i-1}\right\| \leqslant \frac{C_{2}}{N_{k, i}}
$$

from Lemma 5. Putting (8.24), (8.25) and (8.26) together we obtain Point (2), similarly as in Lemma of [5]. For Point (3), we write

$$
\mu_{k, i}-I_{f_{k, i}}=\left(\mu_{k, i}-I_{F_{k, i}}\right)+\left(I_{F_{k, i}}-I_{f_{k, i}}\right)
$$

and conclude thanks to Lemmas 6 and 8 .

We now turn to the proof of the Stage Theorem.

Proof of Theorem 9. From the Step theorem we know that $\left\|\mu_{k, 1}-I_{f_{k, 1}}\right\|=O\left(\frac{1}{N_{k, 1}}\right)$. In order to apply a second step we need to prove that $\rho_{2}\left(D_{k, 2}\right)>0$ over $\mathcal{D}_{k, 2}$. Let $\operatorname{Err}_{k, 1}=\mu_{k, 1}-I_{f_{k, 1}}$. From the definition of the $D_{k, i}$ we have

$$
D_{k, 2}=D_{k, 1}+I_{k, 2}-I_{k, 1}+\operatorname{Err}_{k, 1}-\rho_{1}\left(D_{k, 1}\right) \ell_{1} \otimes \ell_{1}
$$

whence

$$
\rho_{2}\left(D_{k, 2}\right)=\rho_{2}\left(D_{k, 1}\right)+\rho_{2}\left(I_{k, 2}-I_{k, 1}\right)+\rho_{2}\left(\operatorname{Err}_{k, 1}\right) .
$$

- Over $\overline{\mathcal{D}_{k, 0}}$, we have $I_{k, 2}=I_{k, 1}$ and $\rho_{2}\left(D_{k, 1}\right)>0$. Choosing $N_{k, 1}$ such that $\rho_{2}\left(\operatorname{Err}_{k, 1}\right)<$ $\rho_{2}\left(D_{k, 1}\right)$ thus ensures that $\rho_{2}\left(D_{k, 2}\right)>0$.

- Over $\overline{\mathcal{D}_{k, 1} \backslash \mathcal{D}_{k, 0}}$, the sum $\rho_{2}\left(D_{k, 1}\right)+\rho_{2}\left(I_{k, 2}-I_{k, 1}\right)$ is strictly positive. Indeed, the two terms are non-negative, $\rho_{2}\left(D_{k, 1}\right)$ only cancels on the boundary of $\mathcal{D}_{k, 1}$ while $\rho_{2}\left(I_{k, 2}-\right.$ $\left.I_{k, 1}\right)$ is strictly positive on this boundary. Choosing $N_{k, 1}$ such that $\rho_{2}\left(E r r_{k, 1}\right)$ is small enough we ensure that $\rho_{2}\left(D_{k, 2}\right)>0$.

- Over $\mathcal{D}_{k, 2} \backslash \mathcal{D}_{k, 1}$, we have $\operatorname{Err}_{k, 1}=D_{k, 1}=0$ and $\rho_{2}\left(I_{k, 2}-I_{k, 1}\right)>0$ whence $\rho_{2}\left(D_{k, 2}\right)>0$.

We thus apply a second convex integration to obtain $f_{k, 2}=I C\left(f_{k, 1}, \mathcal{D}_{k, 2}, N_{k, 2}\right)$. Similar arguments show that $\rho_{3}\left(D_{k, 3}\right)>0$ provided that $N_{k, 1}^{-1}$ and $N_{k, 2}^{-1}$ are small enough. We can eventually perform a third convex integration to get $f_{k, 3}=I C\left(f_{k, 2}, \mathcal{D}_{k, 3}, N_{k, 3}\right)$.

We now prove point (1) of the theorem. Putting $E r r_{k, i}=\mu_{k, i}-I_{f_{k, i}}$, we first observe that

$$
\operatorname{Err}_{k, i}=\left(I_{f_{k, i-1}}-I_{f_{k, i}}\right)+\rho_{i}\left(D_{k, i}\right) \ell_{i} \otimes \ell_{i}
$$

and

$$
\mu_{k, i}=I_{k, i}-\sum_{j \neq i} \rho_{j}\left(D_{k, i}\right) \ell_{j} \otimes \ell_{j}
$$


Whence for $j \neq i, \rho_{j}\left(\operatorname{Err}_{k, i}\right)=\rho_{j}\left(I_{f_{k, i-1}}-I_{f_{k, i}}\right)$. We also have $\rho_{i}\left(\mu_{k, i}\right)=\rho_{i}\left(I_{k, i}\right)$, so that $\rho_{i}\left(\operatorname{Err}_{k, i}\right)=\rho_{i}\left(I_{k, i}-I_{f_{k, i}}\right)$. We have

$$
\begin{aligned}
D_{k+1,1} & =I_{k+1,1}-I_{f_{k, 3}} \\
& =\left(I_{k+1,1}-I_{k, 1}\right)+\left(I_{k, 1}-I_{f_{k, 1}}\right)+\left(I_{f_{k, 1}}-I_{f_{k, 2}}\right)+\left(I_{f_{k, 2}}-I_{f_{k, 3}}\right) .
\end{aligned}
$$

We deduce

$$
\rho_{1}\left(D_{k+1,1}\right)=\rho_{1}\left(I_{k+1,1}-I_{k, 1}\right)+\rho_{1}\left(\operatorname{Err}_{k, 1}\right)+\rho_{1}\left(\operatorname{Err}_{k, 2}\right)+\rho_{1}\left(\operatorname{Err}_{k, 3}\right)
$$

Note that each $\operatorname{Err}_{k, i}$ vanishes over $\mathcal{D} \backslash \mathcal{D}_{k, 3}$ and can be made arbitrarily small over $\mathcal{D}_{k, 3}$ by a convenient choice of the $N_{k, i}$. Since the $\rho_{i}\left(I_{k+1,1}-I_{k, 1}\right)$ are strictly positive over $\mathcal{D}_{k+1,1}$ we conclude that $\rho_{1}\left(D_{k+1,1}\right)>0$ over $\mathcal{D}_{k+1,1}$. Similar arguments show that $\rho_{2}\left(D_{k+1,1}\right)$ and $\rho_{3}\left(D_{k+1,1}\right)$ are strictly positive over $\mathcal{D}_{k+1,1}$.

We now prove point (2). We write $I_{k, 3}-I_{f_{k, 3}}=\left(I_{k, 3}-I_{k+1,1}\right)+D_{k+1,1}$ and focus on $D_{k+1,1}$. Recall from (8.27) that for $i=1,2,3$,

$$
D_{k, i+1}=D_{k, i}+I_{k, i+1}-I_{k, i}+\operatorname{Err}_{k, i}-\rho_{i}\left(D_{k, i}\right) \ell_{i} \otimes \ell_{i},
$$

where by convention $D_{k, 4}:=D_{k+1,1}$ and $I_{k, 4}:=I_{k+1,1}$. By summation we get

$$
\begin{aligned}
D_{k+1,1} & =D_{k, 1}+\left(I_{k+1,1}-I_{k, 1}\right)-\sum_{i=1}^{3} \rho_{i}\left(D_{k, i}\right) \ell_{i} \otimes \ell_{i}+\sum_{i=1}^{3} \operatorname{Err}_{k, i} \\
& =I_{k+1,1}-I_{k, 1}+\sum_{i=1}^{3} \operatorname{Err}_{k, i}-\rho_{2}\left(D_{k, 2}-D_{k, 1}\right) \ell_{2} \otimes \ell_{2}-\rho_{3}\left(D_{k, 3}-D_{k, 1}\right) \ell_{3} \otimes \ell_{3} .
\end{aligned}
$$

The last two terms $\rho_{2}\left(D_{k, 2}-D_{k, 1}\right) \ell_{2} \otimes \ell_{2}$ and $\rho_{3}\left(D_{k, 3}-D_{k, 1}\right) \ell_{3} \otimes \ell_{3}$ can be bounded as follows. From the definition of $\operatorname{Err}_{k, i}$ we may write

$$
\begin{aligned}
\operatorname{Err}_{k, 1} & =I_{k, 1}-\rho_{2}\left(D_{k, 1}\right) \ell_{2} \otimes \ell_{2}-\rho_{3}\left(D_{k, 1}\right) \ell_{3} \otimes \ell_{3}+D_{k, 2}-I_{k, 2} \\
& =I_{k, 1}-I_{k, 2}+\rho_{1}\left(D_{k, 2}\right) \ell_{1} \otimes \ell_{1}+\rho_{2}\left(D_{k, 2}-D_{k, 1}\right) \ell_{2} \otimes \ell_{2}+\rho_{3}\left(D_{k, 2}-D_{k, 1}\right) \ell_{3} \otimes \ell_{3} .
\end{aligned}
$$

We deduce from (2.3) that $\left|\rho_{2}\left(D_{k, 2}-D_{k, 1}\right)\right| \leqslant \frac{\sqrt{6}}{2}\left(\left\|\operatorname{Err}_{k, 1}\right\|+\left\|I_{k, 2}-I_{k, 1}\right\|\right)$. Similarly,

$$
\operatorname{Err}_{k, 2}=I_{k, 2}-I_{k, 3}+\rho_{1}\left(D_{k, 3}-D_{k, 2}\right) \ell_{1} \otimes \ell_{1}+\rho_{2}\left(D_{k, 3}\right) \ell_{2} \otimes \ell_{2}+\rho_{3}\left(D_{k, 3}-D_{k, 2}\right) \ell_{3} \otimes \ell_{3}
$$

and by summation, we obtain

$$
\operatorname{Err}_{k, 1}+\operatorname{Err}_{k, 2}-\left(I_{k, 1}-I_{k, 3}\right)=(*) \ell_{1} \otimes \ell_{1}+(*) \ell_{2} \otimes \ell_{2}+\rho_{3}\left(D_{k, 3}-D_{k, 1}\right) \ell_{3} \otimes \ell_{3} .
$$

Whence $\left|\rho_{3}\left(D_{k, 3}-D_{k, 1}\right)\right| \leqslant \sqrt{2}\left(\left\|\operatorname{Err}_{k, 1}\right\|+\left\|\operatorname{Err}_{k, 2}\right\|+\left\|I_{k, 3}-I_{k, 1}\right\|\right)$. Back to our initial objective, we can now write

$$
\left\|I_{k, 3}-I_{f_{k, 3}}\right\| \leqslant\left\|I_{k, 3}-I_{k+1,1}\right\|+\left\|D_{k+1,1}\right\| \leqslant \mathcal{I}+\mathcal{E}
$$

where

$$
\mathcal{I}=\left\|I_{k, 3}-I_{k+1,1}\right\|+\left\|I_{k+1,1}-I_{k, 1}\right\|+\frac{\sqrt{6}}{2}\left\|I_{k, 2}-I_{k, 1}\right\|+\sqrt{2}\left\|I_{k, 3}-I_{k, 1}\right\|
$$

and

$$
\mathcal{E}=\sum_{i=1}^{3}\left\|\operatorname{Err}_{k, i}\right\|+\frac{\sqrt{6}}{2}\left\|\operatorname{Err}_{k, 1}\right\|+\sqrt{2}\left(\left\|\operatorname{Err}_{k, 1}\right\|+\left\|\operatorname{Err}_{k, 2}\right\|\right)
$$

By the interpolation formula of the metrics we have over $\mathcal{D}_{k, 0}$ :

$$
\left\|I_{k+1,1}-I_{k, 3}\right\|=\left\|I_{k+1,1}-I_{k, 1}\right\|=\left\|I_{k+1}-I_{k}\right\| \leqslant \tau_{k}^{2}\left\|I_{h}-I_{f_{0}}\right\| \text { and } I_{k, 1}=I_{k, 2}=I_{k, 3} \text {. }
$$


AN EXPLICIT ISOMETRIC REDUCTION OF THE UNIT SPHERE INTO AN ARBITRARILY SMALL BALLDEDICATED TO THE MEMO

It follows that $\mathcal{I} \leqslant 2 \tau_{k}^{2}\left\|I_{h}-I_{f_{0}}\right\|$. Over $\mathcal{D} \backslash \mathcal{D}_{k, 0}$, we have:

$$
\left\|I_{k+1,1}-I_{k, 3}\right\|,\left\|I_{k+1,1}-I_{k, 1}\right\|,\left\|I_{k, 2}-I_{k, 1}\right\|,\left\|I_{k, 3}-I_{k, 1}\right\| \leqslant\left\|I_{h}-I_{f_{0}}\right\| \leqslant \tau_{k-1}^{2}
$$

where the last inequality comes from the choice of the domain $\mathcal{D}_{k, 0}$. We conclude that the inequality

$$
\mathcal{I} \leqslant \frac{C^{2}}{2} \tau_{k-1}^{2} \quad \text { with } \quad C^{2}=2 \max \left(2+\frac{\sqrt{6}}{2}+\sqrt{2}, 2\left\|I_{h}-I_{f_{0}}\right\|\right)
$$

holds over $\mathcal{D}$. By point (3) of the Step Theorem, we can choose $N_{k, 1}, N_{k, 2}$ and $N_{k, 3}$ so that $\mathcal{E} \leqslant \frac{C^{2}}{2} \tau_{k-1}^{2}$. This concludes the proof of point (2).

It remains to prove point (3) of the theorem. We first write

$$
\left\|d f_{k, 3}-d f_{k, 0}\right\| \leqslant \sum_{i=1}^{3}\left\|d f_{k, i}-d f_{k, i-1}\right\| .
$$

From point (2) of the Step Theorem we deduce that

$$
\left\|d f_{k, 3}-d f_{k, 0}\right\| \leqslant \sum_{i=1}^{3} O\left(\frac{1}{N_{k, i}}\right)+2 \sqrt{7} \sum_{i=1}^{3}\left\|\rho_{i}\left(D_{k, i}\right)\right\|^{1 / 2} .
$$

Recall that $\left\|\rho_{1}\left(D_{k, 1}\right)\right\| \leqslant \frac{\sqrt{6}}{2}\left\|D_{k, 1}\right\|=\frac{\sqrt{6}}{2}\left\|I_{k, 1}-I_{f_{k, 0}}\right\|$. For $\rho_{2}\left(D_{k, 2}\right)$ we have by Formula $(8.28)$ and by the subadditivity of the square root:

$$
\begin{aligned}
\left\|\rho_{2}\left(D_{k, 2}\right)\right\|^{1 / 2} & \leqslant\left\|\rho_{2}\left(D_{k, 1}\right)\right\|^{1 / 2}+\left\|\rho_{2}\left(I_{k, 2}-I_{k, 1}\right)\right\|^{1 / 2}+\left\|\rho_{2}\left(\operatorname{Err}_{k, 1}\right)\right\|^{1 / 2} \\
& \leqslant\left(\frac{3}{2}\right)^{1 / 4}\left(\left\|D_{k, 1}\right\|^{1 / 2}+\left\|I_{k, 2}-I_{k, 1}\right\|^{1 / 2}+\left\|\operatorname{Err}_{k, 1}\right\|^{1 / 2}\right) .
\end{aligned}
$$

Arguing as for point (2), we have by a convenient choice of $N_{k, 1}$ and some appropriate constant $C_{1}$

$$
\left\|\rho_{2}\left(D_{k, 2}\right)\right\|^{1 / 2} \leqslant C_{1}\left(\left\|D_{k, 1}\right\|^{1 / 2}+\tau_{k-1}\right) .
$$

A similar computation shows that

$$
\left\|\rho_{3}\left(D_{k, 3}\right)\right\|^{1 / 2} \leqslant C_{2}\left(\left\|D_{k, 1}\right\|^{1 / 2}+\tau_{k-1}\right)
$$

Altogether we get

$$
\left\|d f_{k, 3}-d f_{k, 0}\right\| \leqslant \sum_{i=1}^{3} O\left(\frac{1}{N_{k, i}}\right)+C_{3}\left(\left\|I_{k, 1}-I_{f_{k, 0}}\right\|^{1 / 2}+\tau_{k-1}\right) .
$$

Choosing the $N_{k, i}$ large enough, we eventually get

$$
\begin{gathered}
\left\|d f_{k, 3}-d f_{k, 0}\right\| \leqslant C_{4}\left(\left\|I_{k, 1}-I_{f_{k, 0}}\right\|^{1 / 2}+\tau_{k-1}\right) \quad \text { and } \\
\left\|I_{k, 1}-I_{f_{k, 0}}\right\|^{1 / 2} \leqslant\left\|I_{k, 1}-I_{k-1,3}\right\|^{1 / 2}+\left\|I_{k-1,3}-I_{f_{k, 0}}\right\|^{1 / 2} \leqslant\left\|I_{k, 1}-I_{k-1,3}\right\|^{1 / 2}+C_{5} \tau_{k-2} .
\end{gathered}
$$

where we used point (2) for the last inequality. We also get from the proof of this point that $\left\|I_{k, 1}-I_{k-1,3}\right\|^{1 / 2} \leqslant C_{6} \tau_{k-2}$ with $C_{6}=\max \left(1,\left\|I_{h}-I_{f_{0}}\right\|^{1 / 2}\right)$. We finally conclude that $\left\|d f_{k, 3}-d f_{k, 0}\right\| \leqslant C^{\prime} \tau_{k-1}$.

We end this section by showing that $w_{k, i}$ is nowhere horizontal.

LEMMA 12. If the corrugation numbers are chosen large enough, the vector field $w_{k, i}$ is never horizontal, i.e. $e_{X}$ and $w_{k, i}$ are everywhere independent on $\mathcal{D}_{k, i}$. 
Proof. For conciseness we put $w=w_{k, i}$ and $\mu=\mu_{k, i}$. Let us write $w=X e_{X}+Y e_{Y}$. It is enough to prove that $Y$ is strictly positive. For $i=3$, this condition is trivially satisfied since by definition of $\ell_{3}$ we have on the one hand $\ell_{3}(w)=Y$ (see Section 2) and by definition of $w$ we have on the other hand $\ell_{3}(w)=1$ (see Section 6). For $i=1,2$ we compute

$$
\left(\begin{array}{l}
X \\
Y
\end{array}\right)=\frac{1}{E G-F^{2}}\left(\begin{array}{cc}
G & -F \\
-F & E
\end{array}\right)\left(\begin{array}{l}
\mu_{X} \\
\mu_{Y}
\end{array}\right)
$$

where $\mu_{X}=\mu\left(w, e_{X}\right)$ and $\mu_{Y}=\mu\left(w, e_{Y}\right)$ and $E, F, G$ are the coefficients of the matrix of $\mu$ in the basis $\left(e_{X}, e_{Y}\right)$. We thus want to prove

$$
-F \mu_{X}+E \mu_{Y}>0 .
$$

Recall from Section 5 that $\mu\left(w, v_{i}\right)=0$ and $\ell_{i}(w)=1$. For $i=1, \mu\left(w, v_{1}\right)=0$ implies $\mu_{X}=\mu_{Y}$ while $\ell_{1}(w)=1$ gives $X+Y=\sqrt{2}$. From (8.29) we derive $\mu_{X} \frac{E-2 F+G}{E G-F^{2}}=\sqrt{2}$. Since $E-2 F+G=\mu\left(e_{X}-e_{Y}, e_{X}-e_{Y}\right)>0$ we deduce $\mu_{X}>0$. Condition (8.30) thus reduces to $E>F$. For $i=2$ similar arguments lead to $E>-F$. The two conditions are implied by $E>|F|$. We now prove by induction that this holds true for every $(k, i)$. Recall from Proposition 1 that the $F$ coefficient of the matrix $I_{f_{0}}$ vanishes everywhere. This is also true for $I_{h}$, hence for all the interpolated matrices $I_{k, i}$. In particular, $E>|F|=0$ for all those matrices. Recall from (7.21) that $\mu_{1,1}=I_{f_{0}}+\rho_{1}\left(I_{1,1}-I_{f_{0}}\right) \ell_{1} \otimes \ell_{1}$, whence

$$
\mu_{1,1}=I_{f_{0}}+\frac{1}{2} \rho_{1}\left(I_{1,1}-I_{f_{0}}\right)\left(\begin{array}{ll}
1 & 1 \\
1 & 1
\end{array}\right) .
$$

Since $\rho_{1}\left(I_{1,1}-I_{f_{0}}\right) \geqslant 0$, the inequality $E_{0}>\left|F_{0}\right|=0$ implies $E\left(\mu_{1,1}\right)>\left|F\left(\mu_{1,1}\right)\right|$ (with obvious notations). It follows that each flow line of $w_{1,1}$ joins the south boundary to the north boundary of $\mathcal{D}_{1,1}$. We can thus apply the CI process to build $f_{1,1}$. From Point (3) in the Step theorem we can choose $N_{1,1}$ large enough so that $E\left(I_{f_{1,1}}\right)>\left|F\left(I_{f_{1,1}}\right)\right|$. From the definition of $\mu_{1,2}$ :

$$
\mu_{1,2}=I_{f_{1,1}}+\rho_{2}\left(I_{1,2}-I_{f_{1,1}}\right) \ell_{2} \otimes \ell_{2}=I_{f_{1,1}}+\frac{1}{2} \rho_{2}\left(I_{1,2}-I_{f_{1,1}}\right)\left(\begin{array}{cc}
1 & -1 \\
-1 & 1
\end{array}\right)
$$

we directly obtain $E\left(\mu_{1,2}\right)>\left|F\left(\mu_{1,2}\right)\right|$. Inductively, the same arguments show that for $N_{k, i}$ large enough we have $E\left(\mu_{k, i+1}\right)>\left|F\left(\mu_{k, i+1}\right)\right|$.

\section{From $C^{\infty}$ to $C^{1}$ Fractal structures}

We denote by $\mathbf{v}_{k, i}$ the normalized derivative of $f_{k, i}$ in the direction $v_{i}$ and by $\mathbf{n}_{k, i}$ the unit normal to $f_{k, i}$. We also set $\mathbf{v}_{k, i}^{\perp}:=\mathbf{v}_{k, i} \times \mathbf{n}_{k, i}$. Obviously, there exists a matrix $\mathcal{C}_{k, i} \in S O(3)$ such that

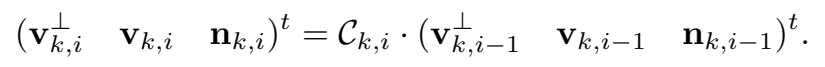

As usual we use the circular convention $*_{k, 0}=*_{k-1,3}$. Here, $\left(\begin{array}{lll}\mathbf{a} & \mathbf{b} & \mathbf{c}\end{array}\right)^{t}$ stands for the transpose of the matrix with column vectors $\mathbf{a}, \mathbf{b}$ and $\mathbf{c}$. The matrix $\mathcal{C}_{k, i}$ encodes the effect of one corrugation on the map $f_{k, i-1}$ and is thus called a corrugation matrix. We set

$$
R(k, i)=\prod_{\ell=k+1}^{\infty}\left(\prod_{j=1}^{3} \mathcal{C}_{\ell, j}\right) \prod_{j=i}^{3} \mathcal{C}_{k, j} .
$$

The Gauss map $\mathbf{n}_{\infty}$ of the limit embedding $f_{\infty}:=\lim _{k \rightarrow+\infty} f_{k, 3}$ can thus be expressed as

$$
\mathbf{n}_{\infty}^{t}=\left(\begin{array}{lll}
0 & 0 & 1
\end{array}\right) \cdot R(k, i) \cdot\left(\mathbf{v}_{k, i-1}^{\perp} \quad \mathbf{v}_{k, i-1} \quad \mathbf{n}_{k, i-1}\right)^{t} .
$$

Together with the description of the asymptotic behaviour of $\mathcal{C}_{k, i}$, the above expression involving an infinite product of matrices was recognized in [4] as a generalized Riesz product and termed 
AN EXPLICIT ISOMETRIC REDUCTION OF THE UNIT SPHERE INTO AN ARBITRARILY SMALL BALLDEDICATED TO THE MEMO

$C^{1}$ fractal structure. In the present case, we have a new phenomenon that arises from the boundary condition.
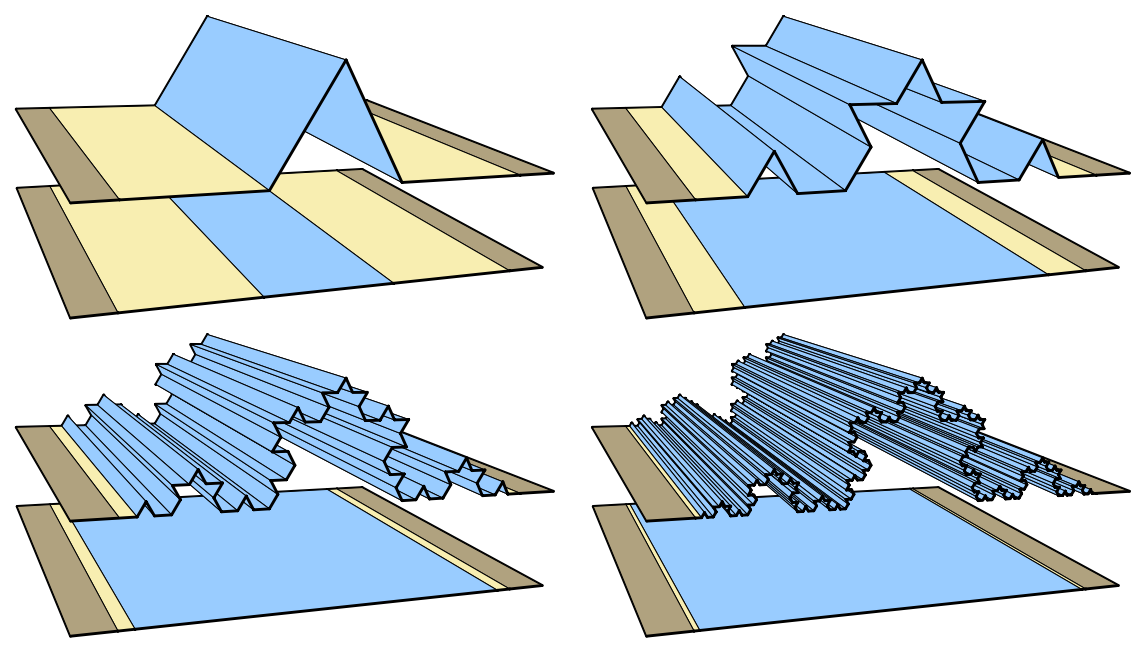

Figure 10. Schematic view of successive corrugations over the sequence of domains.

TheOREM 13 ( $C^{1}$ fractal expansion). The Gauss map $\mathbf{n}_{\infty}$ of the limit embedding $f_{\infty}:=$ $\lim _{k \rightarrow+\infty} f_{k, 3}$ over the domain $\mathcal{D}_{k, i} \backslash \mathcal{D}_{k, i-1}$, where $k \geqslant 1$ and $i \in\{1,2,3\}$, is given by

$$
\mathbf{n}_{\infty}^{t}=\left(\begin{array}{lll}
0 & 0 & 1
\end{array}\right) \cdot R(k, i) \cdot\left(\begin{array}{lll}
\mathbf{v}_{0, i}^{\perp} & \mathbf{v}_{0, i} & \mathbf{n}_{0}
\end{array}\right)^{t}
$$

where $\mathbf{v}_{0, i}$ is the normalized derivative of $f_{0}$ in the direction $v_{i}$, the vector $\mathbf{n}_{0}$ is the unit normal to $f_{0}$, and $\mathbf{v}_{0, i}^{\perp}:=\mathbf{v}_{0, i} \times \mathbf{n}_{0}$.

Proof. The theorem is a direct consequence of Formula (9.31) noting that $f_{k, i-1}=f_{0}$ over $\mathcal{D}_{k, i} \backslash \mathcal{D}_{k, i-1}$.

The $C^{1}$ fractal expansion theorem allows us to describe the transition between the smooth structure of the polar caps and the $C^{1}$ fractal structure of the equatorial belt. At the center of the equatorial belt, the limit Gauss map is extracted from the infinite product of rotation matrices $R(1,1)$ applied to an initial frame. As we get closer to the boundary of $\mathcal{D}$, this infinite product is replaced by its successive remainders $R(k, i)$. As illustrated in Figure 1 moving from the boundary of the polar caps towards the equator, new corrugations arise with larger and larger amplitudes. An analogous phenomenon occurs with the von Koch curve. In the recursive construction from an initial line segment, each step amounts to add a piecewise linear corrugation over a larger and larger central subsegment (See Figure 10). Walking from an endpoint of the resulting von Koch curve towards its center we observe a $C^{0}$ fractal structure with an expansion phenomenon.

\section{CONCLUSION}

Our constructions of a reduced sphere, and previously of an isometric embedding of a flat torus [4], naturally raise the question of their generalization to arbitrary Riemannian surfaces. The constructions are based upon convex integration processes that apply to family of flow lines that foliate pieces of surfaces. As opposed to the torus, the sphere has no global foliation. This explains why we chose to cut the sphere into a central ribbon (topologically, a cylinder) 
and two caps. The initial short embedding of the central ribbon was further chosen so that the isometric default fits into the convex region spanned by three primitive metrics. This is a crucial point in our construction in Section 5. Since both the flow lines and the isometric default change at each CI process, this would a priori impose a new decomposition of the sphere at each step leading to a potentially increasing number of pieces. We resolved this point by proving that we could use a unique decomposition for the whole process, see Lemma 12 and point (1) in the Stage Theorem. Working with a single decomposition greatly simplifies the pasting of the different pieces and brought out the $C^{1}$ fractal expansion phenomenon.

On surfaces of genus two or more one could similarly resort on a decomposition into elementary pieces but the existence of a single decomposition that would fulfill the above conditions on flow lines and isometric default is a real issue. The convex integration process should probably be reviewed or drastically simplified before one could describe an effective construction. Producing isometric embeddings of general Riemannian surfaces remains a challenge.

\section{ACKNOWLEDGEMENT}

The authors thank the anonymous referees for their scrutinous and insightful comments.

\section{REFERENCES}

1. François Apéry, Models of the real projective plane: computer graphics of Steiner and Boy surfaces, Springer, 1987.

2. Yurii F. Borisov, $C^{1, \alpha}$ isometric immersions of Riemannian spaces, Doklady Akademii Nauk SSSR 163 (1965), no. 1, 11.

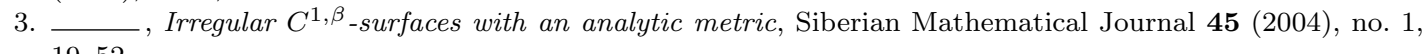
$19-52$.

4. Vincent Borrelli, Saïd Jabrane, Francis Lazarus, and Boris Thibert, Flat tori in three dimensional space and convex integration, Proceedings of the National Academy of Sciences of the United States of America (PNAS) 109 (2012), no. 19, 7218-7223.

5. _ Isometric embeddings of the square flat torus in ambient space, Ensaios Matemáticos 24 (2013), $1-91$.

6. Werner Boy, Über die curvatura integra und die topologie geschlossener flächen, Mathematische Annalen 57 (1903), no. 2, 151-184.

7. Stephan E. Cohn-Vossen, Die verbiegung von flachen im grossen, Fortschr. Math. Wiss 1 (1936), 33-76.

8. Sergio Conti, Camillo De Lellis, and László Székelyhidi Jr, h-principle and rigidity for $C^{1, \alpha}$ isometric embeddings, Nonlinear partial differential equations, Abel Symposia, vol. 7, Springer, 2012, pp. 83-116.

9. Yakov Eliashberg and Nikolai Mishachev, Introduction to the h-principle, Graduate Studies in Mathematics, vol. 48, A.M.S., Providence, 2002.

10. George Francis and John M. Sullivan, Visualizing a Sphere Eversion, IEEE Trans. Vis. and Comp. Graphics 10 (2004), no. 5, 509-515.

11. Mikhail Gromov, Partial differential relations, Springer-Verlag, 1986.

12. Noel J. Hicks, Notes on differential geometry, Math. Studies, Van Nostrand Reinhold, Princeton, NJ, 1965.

13. Norbert Hungerbühler and Micha Wasem, The one-sided isometric extension problem, arXiv preprint arXiv:1410.0232 (2015).

14. Nicolaas H. Kuiper, On $C^{1}$-isometric imbeddings, Indag. Math. 17 (1955), 545-555.

15. Silvio Levy, Making waves. A guide to the ideas behind outside in, AK Peters, Wellesley, MA, 1995.

16. Benoit B Mandelbrot, The fractal geometry of nature, vol. 173, Macmillan, 1983.

17. David Mumford, Caroline Series, and David Wright, Indra's pearls: the vision of felix klein, Cambridge University Press, 2002.

18. John F. Nash, $C^{1}$-isometric imbeddings, Ann. of Math. 60 (1954), no. 3, 383-396.

19. David Spring, Convex integration theory, Monographs in Mathematics, vol. 92, Birkhäuser Verlag, 1998. 
AN EXPLICIT ISOMETRIC REDUCTION OF THE UNIT SPHERE INTO AN ARBITRARILY SMALL BALLDEDICATED TO THE MEMO

E-mail address, Evangelis Bartzos: vangelishowl@yahoo.gr

E-mail address, Vincent Borrelli: borrelli@math.univ-lyon1.fr

E-mail address, Roland Denis: roland.denis@math.univ-lyon1.fr

E-mail address, Francis Lazarus: Francis.Lazarus@gipsa-lab.fr

E-mail address, Damien Rohmer: damien.rohmer@inria.fr

E-mail address, Boris Thibert: Boris.Thibert@univ-grenoble-alpes.fr 